\title{
New and efficient equation-of-motion coupled-cluster framework for core-excited and core-ionized states
}

\author{
Lopez Vidal, Marta; Feng, Xintian; Epifanovsky, Evgeny; Krylov, Anna I.; Coriani, Sonia
}

Published in:

Journal of Chemical Theory and Computation

Link to article, DOI:

10.1021/acs.jctc.9b00039

Publication date:

2019

Document Version

Peer reviewed version

Link back to DTU Orbit

Citation (APA):

Lopez Vidal, M., Feng, X., Epifanovsky, E., Krylov, A. I., \& Coriani, S. (2019). New and efficient equation-ofmotion coupled-cluster framework for core-excited and core-ionized states. Journal of Chemical Theory and Computation, 15(5), 3117-3133. https://doi.org/10.1021/acs.jctc.9b00039

\section{General rights}

Copyright and moral rights for the publications made accessible in the public portal are retained by the authors and/or other copyright owners and it is a condition of accessing publications that users recognise and abide by the legal requirements associated with these rights.

- Users may download and print one copy of any publication from the public portal for the purpose of private study or research.

- You may not further distribute the material or use it for any profit-making activity or commercial gain

- You may freely distribute the URL identifying the publication in the public portal 


\title{
Spectroscopy and Excited States
}

Subscriber access provided by DTU Library

\section{A new and efficient equation-of-motion coupled-cluster framework for core-excited and core-ionized states}

\author{
Marta Lopez Vidal, Xintian Feng, Evgeny Epifanovsky, Anna I. Krylov, and Sonia Coriani
}

J. Chem. Theory Comput., Just Accepted Manuscript • DOI: 10.1021/acs.jctc.9b00039 • Publication Date (Web): 09 Apr 2019

Downloaded from http://pubs.acs.org on April 15, 2019

\section{Just Accepted}

"Just Accepted" manuscripts have been peer-reviewed and accepted for publication. They are posted online prior to technical editing, formatting for publication and author proofing. The American Chemical Society provides "Just Accepted" as a service to the research community to expedite the dissemination of scientific material as soon as possible after acceptance. "Just Accepted" manuscripts appear in full in PDF format accompanied by an HTML abstract. "Just Accepted" manuscripts have been fully peer reviewed, but should not be considered the official version of record. They are citable by the Digital Object Identifier (DOI®). "Just Accepted" is an optional service offered to authors. Therefore, the "Just Accepted" Web site may not include all articles that will be published in the journal. After a manuscript is technically edited and formatted, it will be removed from the "Just Accepted" Web site and published as an ASAP article. Note that technical editing may introduce minor changes to the manuscript text and/or graphics which could affect content, and all legal disclaimers and ethical guidelines that apply to the journal pertain. ACS cannot be held responsible for errors or consequences arising from the use of information contained in these "Just Accepted" manuscripts. 


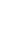

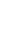

\title{
A new and efficient equation-of-motion
} coupled-cluster framework for core-excited and core-ionized states

\author{
Marta L. Vidal, ${ }^{\dagger}$ Xintian Feng, ${ }^{\ddagger}$ Evgeny Epifanovsky, ${ }^{\complement}$ Anna I. Krylov, ${ }^{\S}$ and \\ Sonia Coriani*, ${ }^{*}$ \\ †DTU Chemistry - Department of Chemistry, Technical University of Denmark, DK-2800, \\ Kongens Lyngby, Denmark \\ $\ddagger$ Department of Chemistry, University of California, Berkeley, California 94720, United \\ States \\ ๑Q-Chem Inc., 6601 Owens Drive, Suite 105 Pleasanton, CA 94588 \\ $\S$ Department of Chemistry, University of Southern California, Los Angeles, California \\ 90089-0482 \\ E-mail: soco@kemi.dtu.dk
}

March 6, 2019

\begin{abstract}
We present a fully analytical implementation of the core-valence separation (CVS) scheme for the equation-of-motion (EOM) coupled-cluster singles and doubles (CCSD) method for calculations of core-level states. Inspired by the CVS idea as originally formulated by Cederbaum, Domcke and Schirmer, pure valence excitations are excluded from the EOM target space and the frozen-core approximation is imposed on
\end{abstract}


the reference-state amplitudes and multipliers. This yields an efficient, robust, practical, and numerically balanced EOM-CCSD framework for calculations of excitation and ionization energies as well as state and transition properties (e.g., spectral intensities, natural transition and Dyson orbitals) from both the ground state and an excited state. The errors in absolute excitation/ionization energies relative to the experimental reference data are of the order of $0.2-3.0 \mathrm{eV}$, depending on the K-edge considered and on the basis set used, and the shifts are systematic for each edge. Compared to a previously proposed CVS scheme where CVS was applied as a posteriori projection only during the solution of the EOM eigenvalue equations, the new scheme is computationally cheaper. It also achieves better cancellation of errors, yielding similar spectral profiles but with absolute core excitation and ionization energies that are systematically closer to the corresponding experimental data. Among the presented results are calculations of transient-state X-ray absorption spectra, relevant for the interpretation of UV-pump/X-ray probe experiments.

\section{Introduction}

By providing tunable high-energy radiation, advanced light sources such as X-ray free electron laser (X-FEL) and synchrotron installations enable a variety of X-ray based spectroscopies. ${ }^{1-3}$ Recent advances in beam quality greatly expanded possible applications of X-rays, giving rise to a proliferation of techniques, including those operating in time-resolved and non-linear regimes. ${ }^{1-5}$ Fundamentally, these spectroscopies exploit electronic transitions involving core orbitals. Since core-level binding energies are characteristic of a species, X-ray absorption and X-ray photo-electron spectroscopies (XAS and XPS, respectively) are powerful techniques for probing electronic structure of atoms and molecules. ${ }^{6}$ The localized nature of core orbitals makes X-ray-based spectroscopies sensitive to local environment. However, just as in the case of VUV-based techniques, ${ }^{7}$ theoretical modeling is required to unambiguously assign spectral features and to relate experimental measurements to molecular 

in developing highly accurate theoretical methods for X-ray spectroscopy and, in particular, for time-resolved XAS, which is proving to be a powerful mean to investigate molecular dynamics. ${ }^{5,8-10}$

Owing to its low computational cost relative to ab initio methods, time-dependent (TD) density functional theory (DFT) has been among the most commonly used techniques for modeling absorption spectra, including X-ray absorption. ${ }^{11}$ However, TDDFT often fails to deliver an accurate description of spectroscopic properties. TDDFT core excitations computed with conventional exchange-correlation functionals, for instance, are grossly underestimated with MAE of the order of $20 \mathrm{eV},{ }^{11}$ and time-independent DFT-based procedures like $\Delta \mathrm{KS}$, transition-potential DFT (TP-DFT) ${ }^{12,13}$ and orthogonality constrained DFT (OCDFT $)^{14,15}$ are found to perform better than TDDFT for X-ray absorption.

Thus, there is an evident need for reliable wave-function-based methods for calculations of XAS spectra of both ground and excited states. Although more expensive, these methods can be systematically improved, thereby yielding to results of controlled accuracy. ${ }^{16}$ The focus of this contribution is on extending coupled-cluster (CC) approaches, ${ }^{17-24}$ which are among the most successful ab initio methods for molecular properties and electronic spectra in the UV-vis region, to absorption and ionization phenomena in the X-ray domain. ${ }^{8,25-28}$

Although ionization or excitations of core electrons superficially appear to be similar to valence transitions, the numerical experiments have shown that direct application of standard approaches to core-level transitions leads to unsatisfactory results, ${ }^{5}$ due to the following essential features of core-ionized and core-excited states. First, these states have open-shell character. ${ }^{29}$ Second, the core-level states lie very high in energy (hundreds of electron volts, depending on the edge). Third, orbital relaxation effects are much more important for core states than for valence states because the outer orbitals are more delocalized and better shielded from the nuclear charge than the tight and localized core orbitals. Fourth, these transitions appear to be much more sensitive to the effect of the environment. ${ }^{30,31}$ Fifth, 
these high-lying states are metastable with respect to electron ejection, ${ }^{32,33}$ i.e., they are Feshbach resonances that can autoionize via two-electron transitions in which one valence electron fills the core hole and a second valence electron is ejected. Thus, they are embedded in the ionization continuum and their description within Hermitian quantum mechanics is problematic.

Multistate methods, such as equation-of-motion Coupled Cluster (EOM-CC), ${ }^{17,18,20,22-24,34}$ can effectively describe multiconfigurational wavefunctions, tackle open-shell character and orbital relaxation effects in one computational scheme. The effect of the environment can be included by using a variety of implicit or explicit solvent models. However, dealing with high-energy interior states, especially with those embedded in autodetaching continuum, is more problematic. Although the Davidson procedure can be modified to solve for the eigenstates dominated by the desired transition (MOM-like ${ }^{35}$ ) or lying within the desired energy range, ${ }^{26,36}$ the convergence and numerical stability are strongly affected by the presence of the continuum. An attempt to compute such states often produces pseudo-continuum states in which one electron occupies the most diffuse orbital. ${ }^{37}$ Because in Hermitian quantum mechanics, the resonances are not represented by a single state, but rather by an increased density of states in the continuum, ${ }^{38}$ it is not possible to obtain converged results by systematically improving the basis set — resonances simply dissolve in the continuum. In moderate bases, resonances appear as isolated states, but their representation in a discretized continuum is inherently prone to numeric instabilities. Although the EOM-CC methods can describe resonances by using complex-scaled and CAP-augmented approaches, ${ }^{33}$ such calculations are much more expensive than regular bound-state calculations. Here we focus on alternative, less expensive approximate methods for modeling spectroscopic properties of the core-level states.

Our strategy for tackling issues due to the continuum nature of the core levels is based on employing the core-valence separation (CVS) approximation proposed by Cederbaum et al. in $1980 .{ }^{39}$ In essence, within the CVS scheme the continuum is projected out, such that 

from the rest of the configurational space, the CVS allows one to extend standard methods for excited and ionized states to the core-level states. ${ }^{28,40-42}$ This separation of full space into the bound part and the continuum is reminiscent of the Feshbach-Fano treatment of resonances. ${ }^{43,44}$ Effectively, CVS results in a decoupling of the highly excited core states from the continuum of valence excitations; it also leads to a significant reduction of the computational costs. The core states can also be decoupled from the continuum by excluding double excitations from the configurational space and treating them perturbatively. ${ }^{30}$ Although numerically stable, this approach suffers from insufficient description of orbital relaxation. ${ }^{30}$ Because the CVS scheme admits selected double excitations that are crucial for describing orbital relaxation, the CVS-EOM/(or, similarly, linear response, LR-)CCSD ${ }^{28}$ ansatz retains sufficient flexibility, resulting in accuracy comparable to that of regular EOM-CCSD/LRCCSD, ${ }^{25,27,45}$ for those (small) cases where full EOM-CCSD or LR-CCSD calculations are feasible. ${ }^{28}$

The analogy with the Feshbach-Fano formalism ${ }^{43,44}$ makes it clear that the CVS ansätze are not approximations to the full parent ansätze — rather, they should be thought of as the diabatized versions of the full methods. For example, CVS-EOM-CCSD is not an approximation to EOM-CCSD, but a diabatized version of it in which the continuum part of the spectra is projected out. Consequently, hierarchical inclusion of higher excitations would ultimately converge to CVS-FCI (full configuration interaction), which is a diabatized representation of the FCI solution. One can recover exact position of the core states (and also their lifetimes) by solving non-Hermitian effective Hamiltonian ${ }^{43}$ obtained using Löwdin projection formalism, ${ }^{46}$ but this is beyond the standard CVS scheme, which has been shown to yield sufficiently reliable estimates of core-level states energies within this diabatic picture. Just as in the practical implementations of the Feshbach-Fano approach, ${ }^{47}$ one needs to define projectors separating the bound part of the spectra from the continuum. This step involves certain arbitrariness: it disappears in the exact limit, ${ }^{43,46}$ but the results for the diabatized 
states depend on the choice of the projector. As explained below, the present version of CVS-EOM-CCSD employs a slightly different projector than the original one. ${ }^{28}$

An implementation of the CVS within EOM-CC and LR-CC theories has been reported by Coriani and Koch. ${ }^{28}$ In their work, the CVS was deployed as an a posteriori projection applied at each iteration of the solution of the EOM/LRCC equations to eliminate excitations that do not involve at least one core electron, whereas the ground state amplitudes and Lagrangian multipliers retained all possible types of excitations. Here, a different strategy is presented: the zero-order wave-function parameters are computed within the frozen-core (fc) approximation and the subsequent EOM/LRCC equations are solved imposing the corevalence separation analytically. The respective state and transition properties such as oscillator strengths, natural transition orbitals (NTOs), Dyson orbitals, exciton descriptors, and so on are obtained from the appropriate densities between different target-state manifolds. Specifically, transition properties can be computed between the ground state and a coreexcited state, as well as between a valence-excited and a core-excited state. This yields an efficient scheme for simulating, e.g., near-edge absorption fine structure (NEXAFS) spectra of both ground and excited states. The latter is required to simulate UV-pump/X-ray-probe experiments and to obtain core-ionization potentials and Dyson orbitals for XPS spectra of medium size molecules. We report illustrative results for NEXAFS and core IEs of all K-edges in neon, water, ammonia, ethylene, vinylfluoride, ozone, adenine, as well as the transient (time-resolved) NEXAFS spectrum of uracil. 


\section{Theory}

\section{$2.1 \quad$ EOM-CCSD}

In the EOM-CC approach, the target-state wave functions are parameterized using the following ansatz: $18,20,22,48$

$$
\left\langle\Psi_{L}\left|=\left\langle\Phi_{0}\left|L e^{-T} ; \quad\right| \Psi_{R}\right\rangle=e^{T} R\right| \Phi_{0}\right\rangle
$$

where $\left|\Phi_{0}\right\rangle$ is a reference Slater determinant and T, $R$ and $L^{\dagger}$ are excitation operators. The excitation operator $T$ is the cluster operator: ${ }^{49}$

$$
\begin{aligned}
T & =\sum_{\mu} t_{\mu} \tau_{\mu}=T_{1}+T_{2}+\cdots+T_{N} \\
T_{1} & =\sum_{i a} t_{i}^{a} a_{a}^{\dagger} a_{i}, \quad T_{2}=\frac{1}{4} \sum_{i j a b} t_{i j}^{a b} a_{a}^{\dagger} a_{i} a_{b}^{\dagger} a_{j}, \quad \ldots
\end{aligned}
$$

with $\tau_{\mu}$ being the excitation operator and $t_{\mu}$ the corresponding cluster amplitudes determined by the $\mathrm{CC}$ equations for the reference state:

$$
\left\langle\Phi_{\mu}\left|\bar{H}-E_{\mathrm{CC}}\right| \Phi_{0}\right\rangle=0 ; \quad E_{\mathrm{CC}}=\left\langle\Phi_{0}|\bar{H}| \Phi_{0}\right\rangle
$$

where $\left\langle\Phi_{\mu}\right.$ |'s represent $\mu$-tuple excited determinants and $\bar{H}$ is the similarity transformed Hamiltonian:

$$
\bar{H}=e^{-T} H e^{T}
$$

The operator $R$ is a general excitation operator:

$$
R=R_{0}+R_{1}+\cdots+R_{n} \equiv \sum_{\mu} r_{\mu} \tau_{\mu}
$$


and $L$ is a de-excitation operator:

$$
L=L_{0}+L_{1}+\cdots+L_{n} \equiv \sum_{\mu} l_{\mu} \tau_{\mu}^{\dagger}
$$

The choice of the reference state and the exact form of $R$ and $L$ depend on the EOM method to be used. ${ }^{20,22}$ Furthermore, in practical calculations the excitation and de-excitation operators must be truncated to some tractable level of excitation. In this work, we focus on the EOM-CCSD family of methods in which the cluster operator $T$ is truncated to single (S) and double (D) excitations, and so are the excitation $R$ and de-excitation $L$ operators.

One of the most obvious applications of EOM theory is the calculation of electronically excited states. ${ }^{18,48}$ In this approach, referred to as EOM-EE (EOM for excitation energies), the optimal reference state is usually the closed-shell ground-state Hartree-Fock determinant and the $R$ and $L$ operators conserve the number of electrons and their spin, taking the following forms:

$$
\begin{gathered}
R^{\mathrm{EE}}=r_{0}+\sum_{i a} r_{i}^{a} a_{a}^{\dagger} a_{i}+\frac{1}{4} \sum_{i j a b} r_{i j}^{a b} a_{a}^{\dagger} a_{i} a_{b}^{\dagger} a_{j}+\ldots \\
L^{\mathrm{EE}}=\sum_{i a} l_{i}^{a} a_{i}^{\dagger} a_{a}+\frac{1}{4} \sum_{i j a b} l_{i j}^{a b} a_{i}^{\dagger} a_{a} a_{j}^{\dagger} a_{b}+\ldots
\end{gathered}
$$

Another common use of EOM is calculations of ionization energies by the EOM-IP (EOM for ionization potentials) method. ${ }^{50-52}$ In this case, the excitation operator changes the number of electrons in the system, assuming the following from:

$$
R^{\mathrm{IP}}=\sum_{i} r_{i} a_{i}+\frac{1}{4} \sum_{i j a} r_{i j}^{a} a_{a}^{\dagger} a_{j} a_{i}+\ldots
$$

The EOM amplitudes $r_{\mu}$ and $l_{\mu}$ are found as stationary points of the EOM functional:

$$
E=\frac{\left\langle\Psi_{L}|H| \Psi_{R}\right\rangle}{\left\langle\Psi_{L} \mid \Psi_{R}\right\rangle}
$$

By applying the bi-variational principle, ${ }^{53,54}$ one arrives to the non-symmetric eigenvalue 
problem:

$$
\left\langle\Phi_{\mu}|\bar{H}-E| R \Phi_{0}\right\rangle=0 ; \quad\left\langle\Phi_{0} L|\bar{H}-E| \Phi_{\mu}\right\rangle=0
$$

where the eigenvectors of the Hamiltonian form a biorthogonal set: ${ }^{18}$

$$
\left\langle\Phi_{0} L_{i} \mid R_{j} \Phi_{0}\right\rangle=\delta_{i j}
$$

Thus, the implementation of EOM-CCSD method boils down to the diagonalization of the effective Hamiltonian $\bar{H}$ in the basis of the reference and of singly and doubly excited determinants, which can be written in matrix form as:

$$
\bar{H}=\left(\begin{array}{ccc}
E_{C C} & \bar{H}_{O S} & \bar{H}_{O D} \\
0 & \bar{H}_{S S} & \bar{H}_{S D} \\
0 & \bar{H}_{D S} & \bar{H}_{D D}
\end{array}\right)
$$

giving rise to the EOM-CCSD right and left eigenvalue equations:

$$
\begin{aligned}
\left(\begin{array}{cc}
\bar{H}_{S S}-E_{C C} & \bar{H}_{S D} \\
\bar{H}_{D S} & \bar{H}_{D D}-E_{C C}
\end{array}\right)\left(\begin{array}{c}
R_{1} \\
R_{2}
\end{array}\right) & =\omega\left(\begin{array}{c}
R_{1} \\
R_{2}
\end{array}\right) \\
\left(\begin{array}{ll}
L_{1} & L_{2}
\end{array}\right)\left(\begin{array}{cc}
\bar{H}_{S S}-E_{C C} & \bar{H}_{S D} \\
\bar{H}_{D S} & \bar{H}_{D D}-E_{C C}
\end{array}\right) & =\omega\left(\begin{array}{ll}
L_{1} & L_{2}
\end{array}\right)
\end{aligned}
$$

where $\omega$ is the energy difference with respect to the reference state.

In practice, Eqs. (14) and (15) are solved iteratively using a generalized Davidson's iterative diagonalization procedure ${ }^{55-57}$ which requires the computation of the right $\sigma$ and left $\tilde{\sigma}$ trial vectors:

$$
\begin{aligned}
& \sigma_{1}=\left(\left[\bar{H}_{S S}-E_{C C}\right] R_{1}\right)_{1}+\left(\bar{H}_{S D} R_{2}\right)_{1} \\
& \sigma_{2}=\left(\bar{H}_{D S} R_{1}\right)_{2}+\left(\left[\bar{H}_{D D}-E_{C C}\right] R_{2}\right)_{2}
\end{aligned}
$$




$$
\begin{aligned}
& \tilde{\sigma}_{1}=\left(L_{1}\left[\bar{H}_{S S}-E_{C C}\right]\right)_{1}+\left(L_{2} \bar{H}_{D S}\right)_{1} \\
& \tilde{\sigma}_{2}=\left(L_{1} \bar{H}_{S D}\right)_{2}+\left(\left[L_{2} \bar{H}_{D D}-E_{C C}\right]\right)_{2}
\end{aligned}
$$

In EOM-CC, molecular properties are often calculated as expectation values, ${ }^{18,54}$ using unrelaxed one-particle density matrices like

$$
\gamma_{p q}=\left\langle\Psi_{L}\left|a_{p}^{\dagger} a_{q}\right| \Psi_{R}\right\rangle
$$

In the present study, we focus on transition properties involving core states, specifically, the oscillator strengths $f$ between the ground state and the core-excited states, and between valence-excited and core-excited states. These can be formulated via contractions of property integral matrices and transition density matrices between state $i$ and $j$, for instance,

$$
f(i \rightarrow j)=\frac{2}{3}\left(\omega_{j}-\omega_{i}\right) \sum_{\alpha=x, y, z} \mu_{\alpha}^{i \rightarrow j} \mu_{\alpha}^{j \rightarrow i}
$$

where

$$
\mu_{\alpha}^{i \rightarrow j}=\sum_{p q} \mu_{p q}^{\alpha} \gamma_{p q}^{i \rightarrow j} ; \quad \mu_{\alpha}^{j \rightarrow i}=\sum_{r s} \mu_{r s}^{\alpha} \gamma_{r s}^{j \rightarrow i}
$$

Above, $\mu^{\alpha}$ refers to a specific Cartesian component of the electric dipole operator and the state $i$ can be either the ground state and an excited state.

The transition density matrices $\gamma_{p q}^{i \rightarrow j}$ and $\gamma_{p q}^{j \rightarrow i}$ are generalizations of Eq. (20), and their specific form in the fc-CVS-EOM-CCSD framework is discussed in the next sections. We here used the so-called unrelaxed EOM transition densities, which differ from the density matrices derived within the LR-CC formalism in that they do not contain terms originating from the amplitudes' response to the external perturbation. In the framework of EOM$\mathrm{CC}$, the amplitude-response terms can also be included by using, for example, a Lagrangian approach, giving rise to the so-called relaxed state and transition density matrices, which 
are identical to those in the LR-CC formalism. ${ }^{17,50,58-61}$ Furthermore, orbital relaxation to the external perturbation is also omitted, as typically done within coupled-cluster response theory. ${ }^{19}$

Transition density matrices describe the changes in electron density upon excitation and can be interpreted as exciton wavefunction ${ }^{62-65}$ when expressed in coordinate space:

$$
\chi\left(r_{e}, r_{h}\right)=\sum_{p q} \gamma_{p q} \phi_{p}\left(r_{e}\right) \phi_{q}\left(r_{h}\right)
$$

where $r_{e}$ and $r_{h}$ denote particle (electron) and hole coordinates, respectively (using $r_{e}=$ $r_{p}=r, \chi$ is reduced to the transition density). Properties of the exciton can be quantified by various expectation values, i.e., exciton size, hole-particle separation and correlation, and so on. These exciton descriptors help to assign state characters. ${ }^{64-66}$ Applying a singular value decomposition (SVD) to the transition matrices yields the so-called NTOs, ${ }^{64-69}$ which can be used to describe the electronic excitations in terms of hole-particle excitons:

$$
\chi\left(r_{e}, r_{h}\right)=\sum_{K} \sigma_{K} \tilde{\phi}_{K}^{e}\left(r_{e}\right) \tilde{\phi}_{K}^{h}\left(r_{h}\right)
$$

where $\tilde{\phi}_{K}^{e}$ and $\tilde{\phi}_{K}^{h}$ are particle and hole orbitals obtained by SVD of $\gamma$ and $\sigma_{K}$ are the corresponding singular values. Usually, only a few $\sigma_{K}$ are non-zero, giving rise to simple interpretation of excited-state characters in terms of one-electron excitations. Note that the sum of $\sigma_{K}^{2}$ is equal to the squared norm of $\gamma$, which provides a simple metric quantifying the one-electron character of the transition, i.e., for pure single excitations, $\|\gamma\|=1$.

\subsection{The fc-CVS-EOM-CCSD method}

The essence of CVS is the separation between the bound and continuum parts of the spectrum, which allows one to compute the bound part of core-level states. The separation depends on the choice of a projector, which is not uniquely defined. This arbitrariness is 
akin to the inherent arbitrariness of diabatic representation of the electronic Hamiltonian: the diabatic states are not uniquely defined, but if the full problem is solved exactly, then the final answer does not depend on a specific choice of diabatic basis. Different flavors of CVS can be defined by different choices of the projectors, and one way to assess the relative merit of a particular choice is by judging the numeric performance of the resulting methods with respect to a chosen reference, either theoretical or experimental. In discussing diabatization schemes, for instance, the magnitude of the coupling elements is often used as a guideline, so better choices of diabatic states yield smaller couplings. The discussion about various CVS schemes has been commonly framed in the literature in terms of the analysis of the matrix elements of the Hamiltonian that couple core-level and valence states and/or core orbitals and valence orbitals. In the discussion below, we follow the second line of reasoning and analyze the difference between different CVS schemes in terms of the Coulomb integrals that are set to zero.

Different renditions of separation between core and valence excitations have been realized for a variety of approximate wavefunction methods as well as for TDDFT, and we refer the interested reader to Ref. 5 for a recent overview. As mentioned in the Introduction, within the CVS-CC framework of Ref. 28, an effective core-valence separation was introduced (as a projection) in the solution of the CC eigenvalue equations since, physically, the core-excited states are energetically far away from the valence excited states.

Already in 1980, Cederbaum et al. ${ }^{39}$ suggested that, since core orbitals are strongly localized in space and energetically are well separated from the valence orbitals, one can decouple core and valence orbitals by zeroing out the respective blocks of the model Hamiltonian, such that the solutions of the Schrödinger equation can be separated into the core and valence domains. In 1985, Barth and Schirmer ${ }^{40}$ implemented this idea within the second-order algebraic diagrammatic construction method $\mathrm{ADC}(2)^{70,71}$ by observing that, in view of negligible coupling between core and valence orbitals, all the following two-electron Coulomb 
integrals are negligible

$$
\begin{array}{r}
\langle I p \mid q r\rangle=\langle p I \mid q r\rangle=\langle p q \mid I r\rangle=\langle p q \mid r I\rangle \approx 0 \\
\langle I J \mid p q\rangle=\langle p q \mid I J\rangle \approx 0 \\
\langle I J \mid K p\rangle=\langle I J \mid p K\rangle=\langle I p \mid J K\rangle=\langle p I \mid J K\rangle \approx 0
\end{array}
$$

where small letters indicate general valence orbitals, and capital letters core orbitals. As a consequence, one can omit the blocks of the ADC propagator matrix $\mathbf{M}$ involving these integrals and therefore decouple pure valence excitations from excitations involving core electrons. This idea was later extended to all other members of the ADC family of propagator methods. ${ }^{41,71-73}$ Since MP2 is an underlying wave-function for the ADC propagator, ${ }^{71}$ omitting the integrals in Eq. (25) immediately corresponds to using the frozen-core approximation for the MP2 ground state energy.

If the above criterion of negligible Coulomb integrals is applied within the CCSD ansatz in the canonical basis, ${ }^{74}$ the resulting CCSD energy and the amplitude equations for the "valence-only" amplitudes $t_{i_{v}}^{a}$ and $t_{i_{v} j_{v}}^{a b}$ are identical to those obtained within the frozen-core approximation - that is, assuming that all ground-state amplitudes and multipliers where at least one occupied index refers to a core orbital are zero.

Inspired by this observation, we here propose to use the frozen core approximation during the determination of the ground-state amplitudes $t_{\mu}$ and Lagrangian multipliers $\lambda_{\mu}$ while constraining the excitation/ionization operators $R$ and $L$ to involve at least one core orbital, which introduces restrictions on the left and right EOM equations, and on the respective density matrices. We name the resulting approach as fc-CVS-EOM-CCSD method.

The programmable expressions for obtaining the right and left excitation vectors within the fc-CVS-EOM-CCSD methods have been derived from Ref. 54 and for the density matrices from Ref. 60 and can be found in the Supplementary Information.

As an illustrative example, we show below how the expression of the right linearly trans- 
formed vector elements $\sigma_{i}^{a}$ is modified for the fc-CVS case. The general expression for $\sigma_{i}^{a}$ is 54

$$
\sigma_{i}^{a}=\sum_{b} F_{a b} r_{i}^{b}-\sum_{j} F_{i j} r_{j}^{a}-\sum_{j b} I_{i b j a}^{1} r_{j}^{b}+\sum_{j b} F_{j b} r_{i j}^{a b}-\frac{1}{2} \sum_{j k b} I_{j k i b}^{6} r_{j k}^{a b}-\frac{1}{2} \sum_{j b c} I_{j a b c}^{7} r_{i j}^{b c}
$$

with

$$
\begin{aligned}
& F_{i a}=f_{i a}+\sum_{j b} t_{j}^{b}\langle i j \| a b\rangle \\
& F_{i j}=f_{i j}+\sum_{a} t_{i}^{a} f_{j a}+\sum_{k a} t_{k}^{a}\langle j k \| i a\rangle+\sum_{k a b} t_{i}^{a} t_{k}^{b}\langle j k \| a b\rangle+\frac{1}{2} \sum_{k b c} t_{j k}^{a c}\langle j k \| b c\rangle \\
& F_{a b}=f_{a b}-\sum_{i} t_{i}^{a} f_{j a}-\sum_{i c} t_{i}^{c}\langle i a \| b c\rangle+\sum_{i j c} t_{i}^{c} t_{j}^{a}\langle i j \| b c\rangle-\frac{1}{2} \sum_{j k c} t_{j k}^{a c}\langle j k \| b c\rangle \\
& I_{i a j b}^{1}=\langle i a \| j b\rangle-\sum_{k} t_{k}^{b}\langle j k \| i a\rangle-\sum_{c} t_{i}^{c}\langle j b \| a c\rangle+\sum_{k c} t_{i}^{c} t_{k}^{b}\langle j k \| a c\rangle-\sum_{k c} t_{i k}^{b c}\langle j k \| a c\rangle \\
& I_{i j k a}^{6}=\langle i j \| k a\rangle-\sum_{c} t_{k}^{c}\langle i j \| a c\rangle \\
& I_{i a b c}^{7}=\langle i a \| b c\rangle-\sum_{j} t_{j}^{a}\langle i j \| b c\rangle
\end{aligned}
$$

It is convenient to further split the occupied orbitals $(i, j, k, l, \ldots)$ into the two sub-blocks: occupied valence labeled with an additional $v$ subindex, $\left(i_{v}, j_{v}, k_{v}, l_{v}, \ldots\right)$, and occupied core orbitals denoted by a capital letter $(I, J, K, L, \ldots)$. Our fc-CVS scheme then entails: $(i)$ reducing the set of occupied orbitals to only the core ones in the excitation process and, $(i i)$ freezing the core orbitals in optimization of the ground state $\mathrm{CC}$ wavefunction parameters (amplitudes and multipliers). Hence, by restricting the EOM excitations to core excitations only, all terms involving only valence excitations disappear in the equations above. The frozen-core approximation further simplifies the equations since the terms in Eqs. (27) involving ground-state amplitudes vanish for the core orbitals, or, in other words, only ground state amplitudes with valence occupied orbitals are retained in the fc-CVS case.

The fc-CVS expression for the linearly transformed trial vector in Eq. (26) thus reads as 
follows:

$$
\begin{aligned}
\sigma_{I}^{a}= & \sum_{b} F_{a b} r_{I}^{b}-\sum_{J} F_{I J} r_{J}^{a}-\sum_{J b} I_{I b J a}^{1} r_{J}^{b}+\sum_{J b} F_{J b} r_{I J}^{a b}+\sum_{j_{v} b} F_{j_{v} b} r_{I j_{v}}^{a b} \\
& -\sum_{J k_{v} b}\left\langle J k_{v}|| I b\right\rangle r_{J k_{v}}^{a b}-\frac{1}{2} \sum_{J K b}\langle J K \| I b\rangle r_{J K}^{a b}-\frac{1}{2} \sum_{J b c} I_{J a b c}^{7} r_{I J}^{b c}-\frac{1}{2} \sum_{j_{v} b c} I_{j_{v} a b c}^{7} r_{I j_{v}}^{b c}
\end{aligned}
$$

$F_{i_{v} a}=f_{i_{v} a}+\sum_{j_{v} b} t_{j_{v}}^{b}\left\langle i_{v} j_{v} \| a b\right\rangle$

$I_{I a J b}^{1}=\langle I a|| J b\rangle-\sum_{k_{v}} t_{k_{v}}^{b}\left\langle J k_{v}|| I a\right\rangle$

$I_{I a b c}^{7}=\langle I a \| b c\rangle-\sum_{j_{v}} t_{j_{v}}^{a}\left\langle I j_{v} \| b c\right\rangle$

We followed the same strategy to derive the expressions for other linearly transformed vector blocks and for the (transition) density matrices; all programmable expressions are given in the Supplementary Information.

We emphasize that we only used the integral screening as per Eq. (25) to justify the use of the frozen-core approximation in the determination of the ground-state CC wavefunction parameters and to write the effective Hamiltonian as given in Eq. (13). The linearly transformed vectors used to determine the target state energies and the transition properties are derived only invoking the frozen-core condition on the ground state parameters while restricting the EOM excitations to core excitations (the latter referred to as the CVS con$\left.\operatorname{dition}^{28}\right)$. Therefore, some terms in the intermediates still contain such integrals. Although small, these terms are found to further slightly improve the flexibility of our ansatz to address, via correlation, the relaxation effects that follow core excitation. Strictly speaking, 
the complete neglect of the integrals in Eq. 25 defines yet another variant of a CVS scheme, which will not be discussed in the present study.

We conclude this section by discussing size-extensivity of our fc-CVS-EOM-CCSD model. As explained, for instance, in Refs. 75 and 76, size-extensivity of the total energy and sizeintensivity of the excitation energies is guaranteed if the singles and doubles block of the first column of the matrix representation of the effective Hamiltonian $\bar{H}$ are zero, as per Eq. (13). The blocks in question contain the amplitude-constraint terms

$$
\Omega_{\mu}=\left\langle\Phi_{\mu}|\exp (-T) H \exp (T)| \Phi_{0}\right\rangle
$$

where, for CCSD, $\left\langle\Phi_{\mu}\right|$ is either a single or a double-excitation determinant with respect to the reference Slater determinant $\left|\Phi_{0}\right\rangle$, see Eq. (3).

Obviously, in fc-CVS-EOM-CCSD

$$
\begin{gathered}
\Omega_{i_{v}}^{a}=\left\langle\Phi_{i_{v}}^{a}|\exp (-T) H \exp (T)| \Phi_{0}\right\rangle=0 \\
\Omega_{i_{v} j_{v}}^{a b}=\left\langle\Phi_{i_{v} j_{v}}^{a b}|\exp (-T) H \exp (T)| \Phi_{0}\right\rangle=0
\end{gathered}
$$

since these terms are identical to the valence-only amplitude equations obtained by application of the frozen-core approximation. As for the remaining terms, in which the occupied indices are either a core $I$, a core $J$ or both, let us consider, for instance, the elements $\Omega_{I}^{a}$. In the frozen-core approximation, they become:

$$
\begin{aligned}
\Omega_{I}^{a} & =\left\langle\Phi_{I}^{a}|\exp (-T) H \exp (T)| \Phi_{0}\right\rangle \\
& =f_{a I}-\sum_{k_{v}} f_{k_{v}} t_{k_{v}}^{a}+\sum_{k_{v} c}\left\langle k_{v} a \| c I\right\rangle t_{k_{v}}^{c}-\frac{1}{2} \sum_{k_{v} l_{v} c}\left\langle k_{v} l_{v} \| c I\right\rangle t_{k_{v} l_{v}}^{c a}-\sum_{k_{v} l_{v}}\left\langle k_{v} l_{v} \| c I\right\rangle t_{k_{v}}^{c} t_{l_{v}}^{a}
\end{aligned}
$$

In the canonical basis, the respective Fock-matrix terms are zero. If one invokes the negligible integrals conditions as per Eq. (25), these blocks would become exactly zero. Similar arguments apply for the blocks $\Omega_{I j_{v}}^{a b}$ and $\Omega_{I J}^{a b}$. We note in passing that one can alternatively 


\section{Computational details}

We implemented the fc-CVS-EOM-CC method in the Q-Chem electronic structure package ${ }^{77,78}$ using the libtensor library. ${ }^{79}$ The geometries of $\mathrm{H}_{2} \mathrm{O}, \mathrm{NH}_{3}, \mathrm{CO}, \mathrm{C}_{2} \mathrm{H}_{4}, \mathrm{C}_{2} \mathrm{H}_{3} \mathrm{~F}$ and $\mathrm{O}_{3}$ were optimized at the fc-CCSD(T)/cc-pVQZ level using CFOUR. ${ }^{80}$ For 9H-adenine, we considered both planar and non-planar structures taken from the literature. The non-planar structure was optimized at the fc-RI-MP2/cc-pVTZ level, ${ }^{81}$ whereas the planar structure was optimized at the B3LYP/cc-pVTZ level. ${ }^{82}$ In the TR-NEXAFS simulations of uracil, we used different structures: an optimized MP2/cc-pVTZ ground-state structure, a groundstate structure and an $\mathrm{S}_{1}$ minimum both optimized at the SF-BH\&HLYP/6-31+G(d,p) level from Ref. 83, and two stationary-point (i.e., zero-gradient) structures for the $\mathrm{S}_{1}$ and $\mathrm{S}_{2}$ excited states obtained at the EOM-CCSD/aug-cc-pVDZ level of theory. All structures were 
planar or almost planar.

In the (TR-)NEXAFS calculations, we considered several different basis sets, and results are here reported for Pople's 6-311++G** (pure $d$ functions) and Dunning's aug-cc-pVTZ and aug-cc-pCVTZ sets. In selected cases, the basis sets were further augmented with uncontracted Rydberg-type functions whose exponents were computed according to the prescription of Kaufmann et al. ${ }^{84}$ Using such system-specific Rydberg functions affords a more compact description of Rydberg states than an alternative brute-force strategy of adding additional diffuse sets with even-tempered exponents. ${ }^{85}$

To analyze the EOM states (i.e., the extent of Rydberg character), we considered, for a few selected cases, the spatial extent of the respective wavefunctions as well as the size of the particle NTO of a transition. ${ }^{69}$ Both approaches deliver similar information, however, when using the former, one needs to consider the difference between the expectation value of the second moment of charges $\left\langle r^{2}\right\rangle$ of the target EOM state and the CCSD reference ${ }^{85}$ because the size of the electronic wave function depends on the system size.

Experimental data were taken from: Ref. 86 for adenine, Ref. 87 for Neon, Ref. 88 for $\mathrm{H}_{2} \mathrm{O}$ and $\mathrm{NH}_{3}$, Ref. 89 and 90 for CO, Ref. 91 for $\mathrm{C}_{2} \mathrm{H}_{4}$ and $\mathrm{C}_{2} \mathrm{H}_{3} \mathrm{~F}$ and Ref. 92 for $\mathrm{O}_{3}$. The experimental NEXAFS spectrum of uracil is from Ref. 93. All experimental spectra were digitized from the original references using WebPlotDigitizer. ${ }^{94}$ The spectra were generated using a Python script and NTOs were visualized using MOLDEN. ${ }^{95}$ The CVS-LR-CCSD results we compare with were obtained using a development version of Dalton. ${ }^{96}$

\section{Results and discussion}

\subsection{Near-Edge Absorption Spectroscopy and core ionization en- ergies}

To test the performance of our method, we first considered the neon atom. Table S4 reports the computed excitation energies, oscillator strengths and IEs with three different basis sets, 
all supplemented with Rydberg-type functions (with $n=2.5-4.5$ ); the corresponding spectra are shown in Fig. 1. As the atomic NEXAFS spectra are due to transitions from the $1 \mathrm{~s}$ orbital to Rydberg states, ${ }^{6}$ the inclusion of Rydberg-type functions (or, alternative, a large set of diffuse functions) is mandatory in order to reproduce the Rydberg progression of peaks in the experimental spectrum. ${ }^{27}$ The extent of Rydberg character in Neon is quantified in Table S5. Table S4 and Fig. 1 also show the results obtained with the CVS-LR-CCSD method in the largest of the three selected basis sets.

The NEXAFS spectra have been shifted, along with the IEs, to align with the first peak of the experimental NEXAFS spectrum (estimated to be at $867.10 \mathrm{eV}$ ). After the shift, the computed peaks match the experimental ones almost perfectly. Of the three sets, Dunning's aug-cc-pCVTZ (+Rydberg) yields the smallest absolute shift from experiment $(+0.19 \mathrm{eV})$, followed by $-0.43 \mathrm{eV}$ of the aug-cc-pVTZ (+Rydberg) set, versus $-0.84 \mathrm{eV}$ of Pople's 6$311+\mathrm{G}^{* *}(+\mathrm{Rydberg})$. The spectral profiles in the two Dunning sets overlap completely after the alignment.

With respect to previously reported CCSD results, ${ }^{27}$ obtained in the aug-cc-pCVTZ basis supplemented with Rydberg functions using the Lanczos algorithm with all electrons correlated (i.e., no CVS), the absolute shift from experiment in the fc-CVS-EOM-CCSD method is lower and has an opposite sign $(+0.19 \mathrm{eV}$ versus $-1.1 \mathrm{eV})$. The shift is also smaller than the one obtained using the CVS-LR-CCSD approach of Ref. 28, whereas the spectral profiles are practically the same, as it can be appreciated from both the results in Table S4 and in Fig. 1. We also draw the reader's attention to Table S2, where the fc-CVSEOMEE-CCSD results for Neon, as well as for a few molecular systems, are compared with the corresponding CVS-LR-CCSD results taken from Ref. 28. 


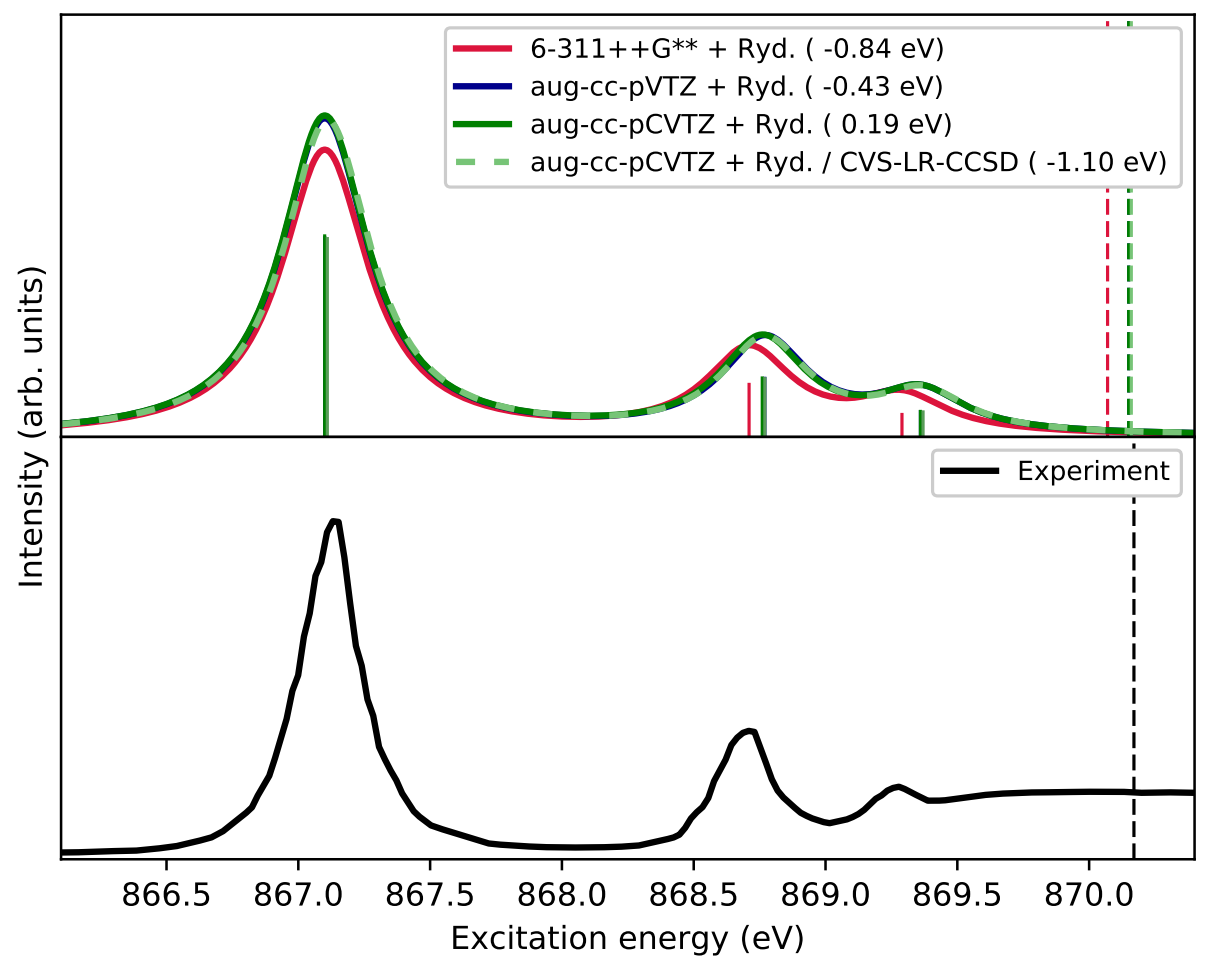

Figure 1: Neon. fc-CVS-EOMEE-CCSD X-ray absorption spectra obtained by convolution of the computed excitation energies and oscillator strengths with a Lorentzian function (FWHM $=0.4 \mathrm{eV}$ ). The experimental spectrum was digitized from Ref. 87. The vertical dashed lines correspond to the core ionization energies. The experimental IE is $870.17 \mathrm{eV}$. The energy shifts required to align the NEXAFS profiles in each basis set with the experimental one are indicated in parenthesis. The computed IEs have been shifted by the same amount as used to align the NEXAFS profiles. The (shifted) NEXAFS spectrum and IE obtained using the CVS-LR-CCSD method ${ }^{28}$ for one basis set are also shown.

The NEXAFS and IE values of $\mathrm{H}_{2} \mathrm{O}$ are reported in Table S6, with the corresponding spectra shown in Fig. 2. The upper panel of Fig. 2 shows the spectra for the chosen basis sets without Rydberg-type functions, whereas the two middle panel show those obtained including the Rydberg-type functions. The third panel in particular compares the spectra and IEs yielded by our fc-EOM-CCSD method and the CVS-LR-CCSD method. Besides an overall shift (taking the value $534.0 \mathrm{eV}$ as reference for the experimental first peak maximum, which varies slightly for the three bases, the separation between the two first peaks is practically the same, whereas huge differences are observed for the other bands known to have Rydberg 
2

3

4

5

6

7

8

9

10

character. Both relative intensity and position of the third band and the following ones are strongly overestimated in the bases without Rydberg functions. Table S5 shows the sizes of particle NTOs and the $\left\langle r^{2}\right\rangle$ values (i.e., the second moments of charge density), clearly revealing Rydberg character of certain transitions.

Also for $\mathrm{H}_{2} \mathrm{O}$, the energy shifts required to realign with the experimental spectrum are smaller than those obtained using CVS-LR-CCSD ${ }^{28}$ see third panel of Fig. 2, as well as Table S7 and Table S2 in the SI. The spectral profiles, on the other hand, are basically identical.

Remarkably, in the aug-cc-pCVTZ basis the shift is smaller that in the aug-cc-pVTZ basis, whereas the reverse trend has been observed using the CVS-LR approach of Ref. 28. Thus, the current approach shows a systematic improvement (in terms of deviation from experiment) of the results with respect to the basis set increase. 


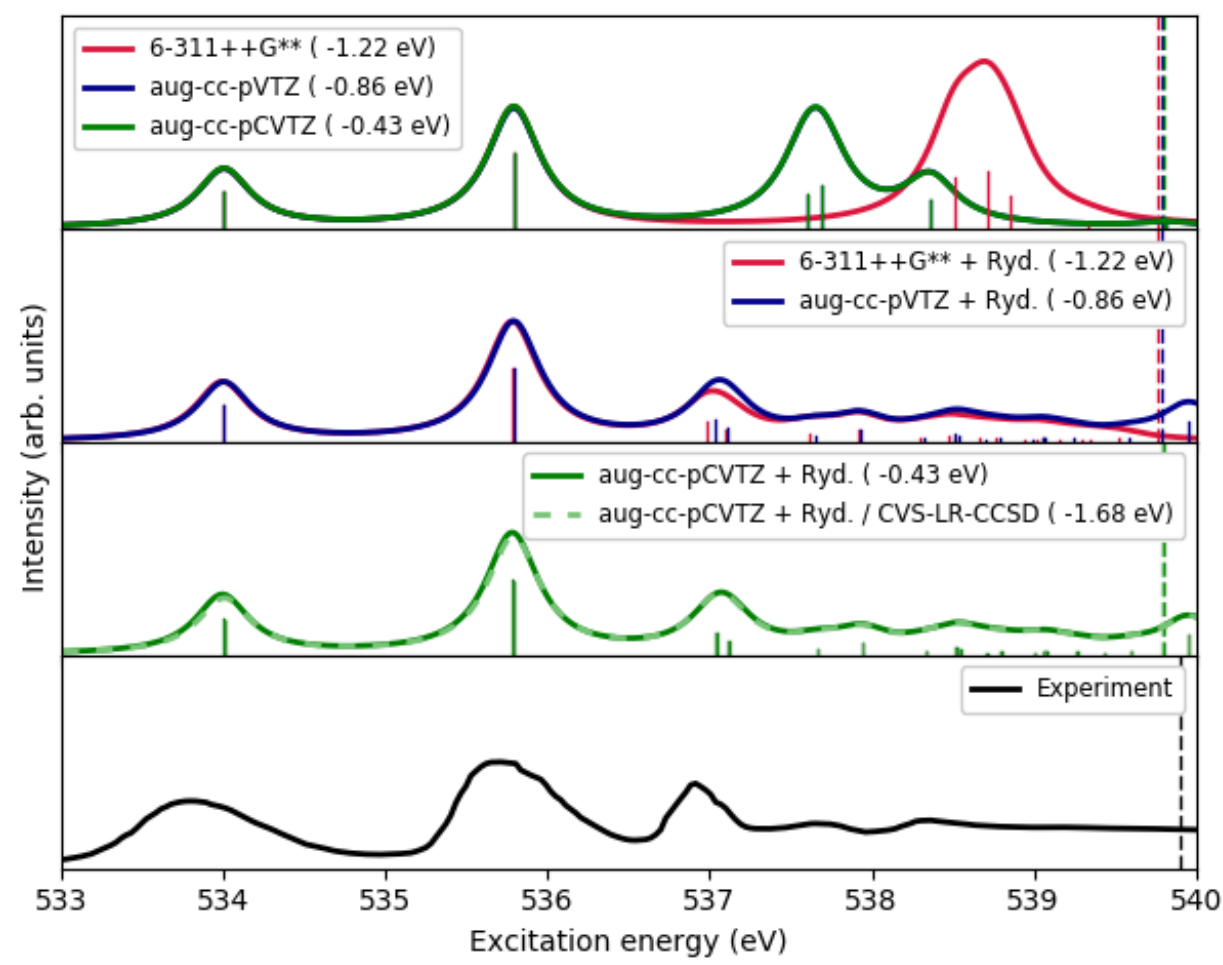

Figure 2: Water. fc-CVS-EOMEE-CCSD O-edge X-ray absorption spectra obtained by convolution of the spectral data in Table S6 with a Lorentzian function $(\mathrm{FWHM}=0.4 \mathrm{eV})$. The experimental spectrum (0.12 eV resolution) was digitized from Ref. 88. Dashed vertical lines correspond to the IEs. The energy shifts required to align the NEXAFS profiles in each basis set with the experimental one are indicated in parenthesis. The computed IEs have been shifted by the same amount as used to align the NEXAFS profiles.

Another system whose gas-phase NEXAFS is dominated by Rydberg states is $\mathrm{NH}_{3}$. Fig. 3 shows the computed spectra; the raw data are in Table S8. The spectra were aligned with respect to the peak maximum of the first experimental band, estimated at $400.53 \mathrm{eV}$. As for the previous systems, the Dunning basis shows a smaller shift compared to the experimental peaks $(-0.68 \mathrm{eV}$ vs $-1.04 \mathrm{eV})$. Neither Pople's 6-311++G** nor Dunning's aug-cc-pVTZ can correctly reproduce the third and higher bands without the inclusion of additional diffuse functions. Rydberg character of these bands is clearly revealed by the data in Table S5. As in the previous two cases, the overall shift of the fc-CVS-EOMEE-CCSD spectra from the experimental one is smaller than obtained with the CVS-LR-CCSD scheme, ${ }^{28}$ see also 
Table S2.

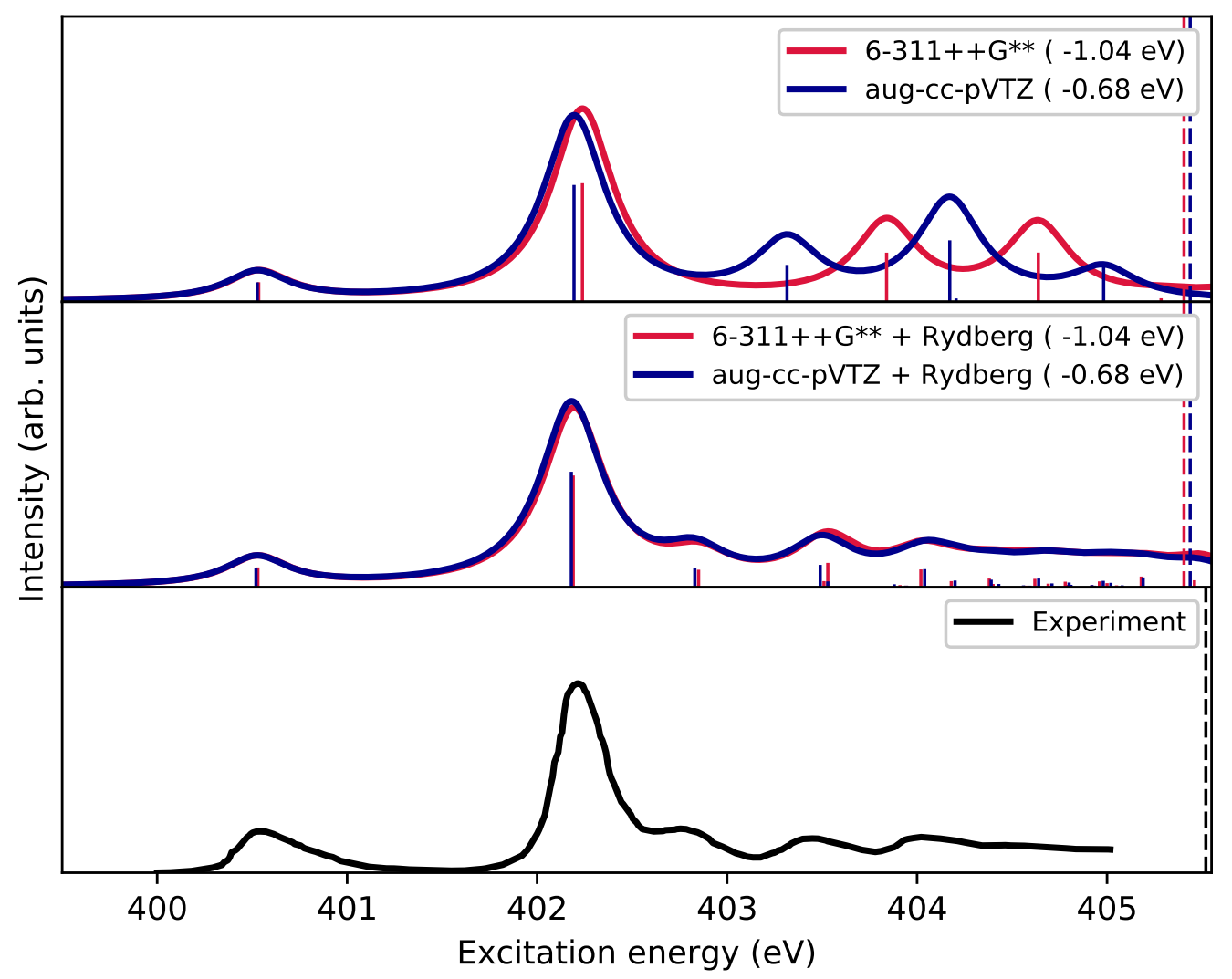

Figure 3: Ammonia. fc-CVS-EOMEE-CCSD N-edge X-ray absorption spectra obtained from convolution of the spectral data in Table S8 with a Lorentzian function $(\mathrm{FWHM}=0.4 \mathrm{eV})$. The experimental spectrum (0.2 eV resolution) was digitized from Ref. 88. Dashed vertical lines indicate the IEs. The energy shifts required to align the NEXAFS profiles in each basis set with the experimental one are indicated in parenthesis. The computed IEs have been shifted by the same amount as used to align the NEXAFS profiles.

Table S10 presents the spectral data for $\mathrm{C}$ and $\mathrm{O}$ edges of carbon monoxide and the corresponding spectra are shown in Fig. 4. The two upper panels in the figure show the main NEXAFS bands, experimentally observed in between 286.5 and $289.0 \mathrm{eV}$ for carbon and in between 533 and $537 \mathrm{eV}$ for oxygen. The middle and bottom panels of Fig. 4 show the (much weaker) peaks observed at higher frequencies below the ionization limit.

The position of the dominant C-edge $1 s \rightarrow \pi^{*}$ band is overestimated by $0.50 \mathrm{eV}$ in the $6-311++\mathrm{G}^{* *}+$ Rydberg basis set, and underestimated by $0.05 \mathrm{eV}$ in the aug-cc-pVTZ + 
Rydberg basis. The O-edge $1 s \rightarrow \pi^{*}$ band is overestimated by about $1.16 \mathrm{eV}$ in Pople's set, and by $0.66 \mathrm{eV}$ in Dunning's basis. The additional features of the main experimental bands are due to the vibronic progression, which is not included in our calculations. The overall shifts are significantly smaller than obtained with the CVS-LR-CCSD scheme, ${ }^{28}$ see also Table S2.

Upon alignment of the computed spectra with the main peak of the experimental ones, the Rydberg transitions are still slightly misaligned, see mid panels of Fig. 4. Nonetheless, all weaker $3 s \sigma, 3 p \pi, 3 p \sigma, 3 d \pi, 4 s \sigma$, and $4 s \pi$ transitions can be identified in the computed spectra of each edge, although once again without their finer vibronic progressions. The assignments can be verified by realignment of the first peak of the first progression, as shown in the bottom panels of Fig. 4 . 

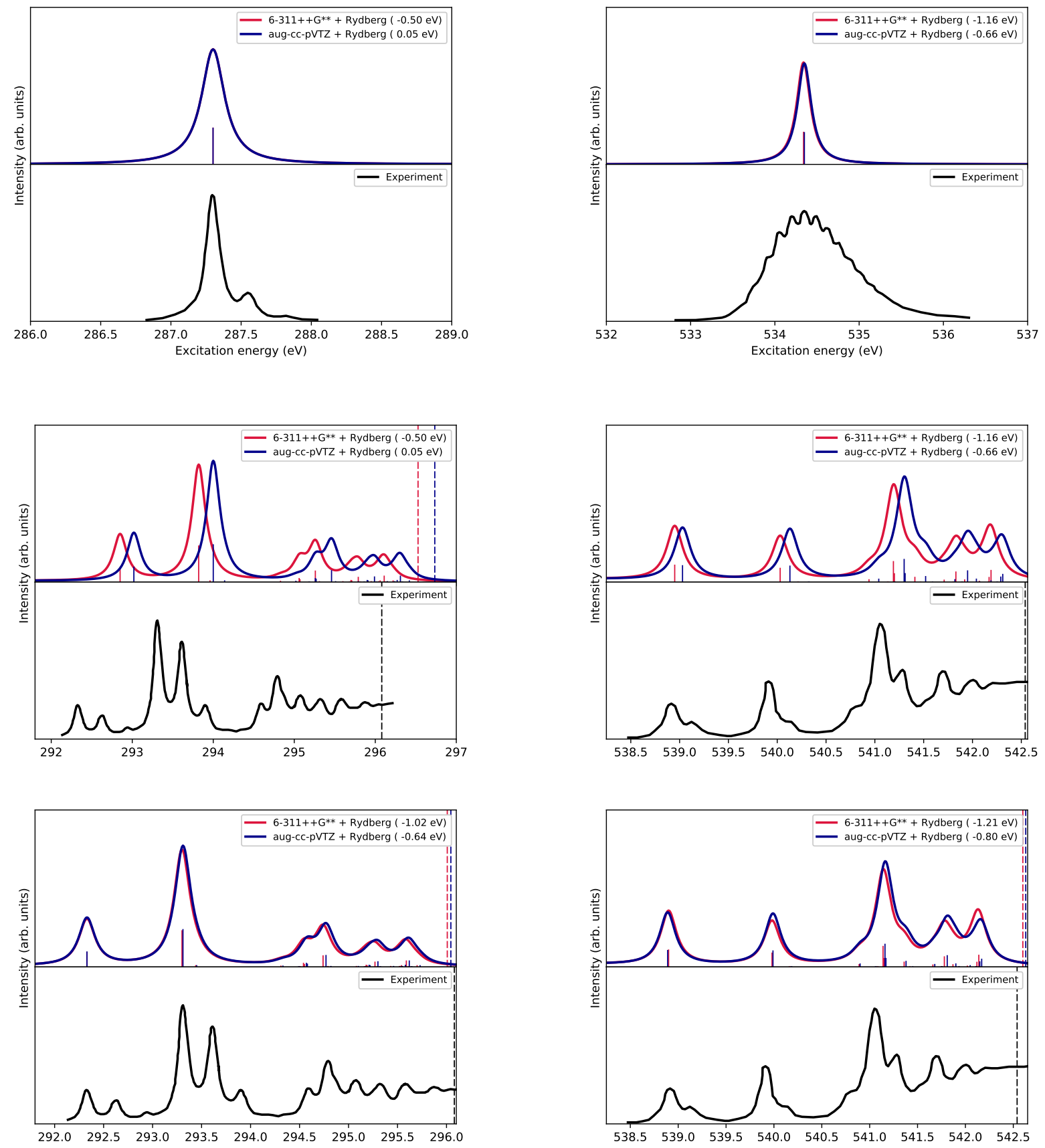

C K-edge

O K-edge

Figure 4: Carbon monoxide. fc-CVS-EOMEE-CCSD C K-edge (left) and O K-edge (right) $\mathrm{X}$-ray absorption spectra, obtained by convolution of the computed excitation energies and oscillator strengths with a Lorentzian function $(\mathrm{FWHM}=0.2 \mathrm{eV})$. The upper panels show the main $1 s \rightarrow \pi^{*}$ band, the mid and bottom panels the band progressions of the weaker $1 s \rightarrow 3 s \sigma, 3 p \pi, 3 d \pi, 4 s \sigma$, and $4 s \pi$ transitions. The experimental spectra ( $0.03 \mathrm{eV}$ resolution at the $\mathrm{C}$ K-edge and $0.07 \mathrm{eV}$ resolution at the O K-edge) were digitized from Ref. 89. Vertical dashed lines correspond to the IEs. 
Fig. 5 reports the computed spectra of ethylene obtained by convolution of the spectral data in Table S11. In this case, the Rydberg functions also improve the description of the higher energy region approaching the ionization limit (third experimental band ${ }^{91}$ ). The second band in the experimental spectrum corresponds to three excitations in the computed spectra. The overall shift is $0.44 \mathrm{eV}$ in the aug-cc-pVTZ(+Rydberg) set and $0.90 \mathrm{eV}$ for Pople's 6-311++G** (+Rydberg) set. The overall shifts are smaller than obtained with the CVS-LR-CCSD scheme 28, see also Table S2. Upon realignment with respect to the $1 s \rightarrow \pi^{*}$ absorption energy, the IE obtained with Pople's set is slightly underestimated compared to the experimental IEs.

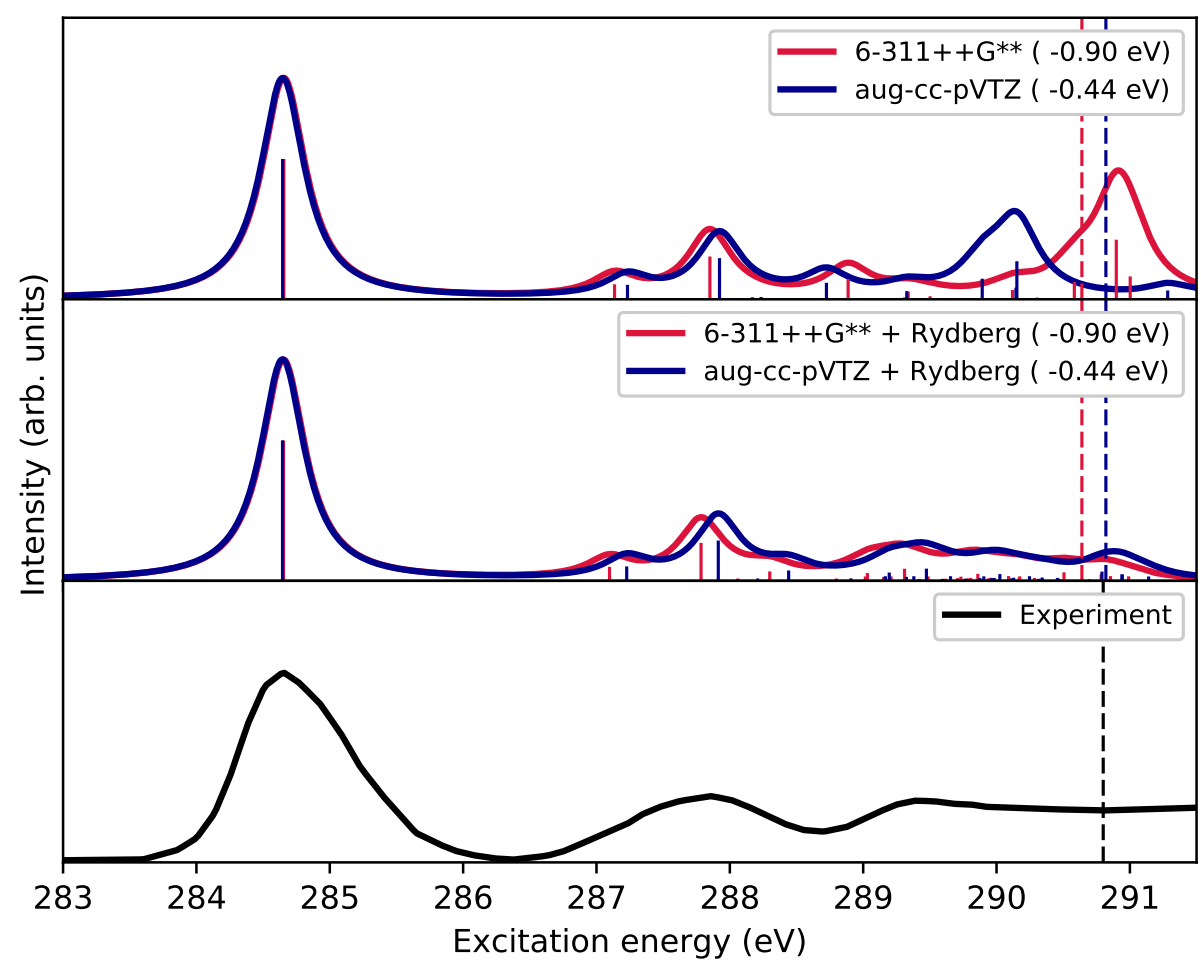

Figure 5: Ethylene. fc-CVS-EOMEE-CCSD X-ray absorption spectra by Lorentzian broadening $(\mathrm{FWHM}=0.4 \mathrm{eV})$ of the computed excitation energies and oscillator strengths. The experimental spectrum (0.6 eV resolution) was digitized from Ref. 91. The vertical dashed lines correspond to the IEs. The energy shifts required to align the NEXAFS profiles in each basis set with the experimental one are indicated in parenthesis. The computed IEs have been shifted by the same amount as used to align the NEXAFS profiles. 
Fig. 6 shows the computed $\mathrm{X}$-ray spectra at the $\mathrm{C}$ edge in vinylfluoride $\left(\mathrm{CH}_{2} \mathrm{CHF}\right)$; the raw data are given in Table S12. The computed spectra were shifted to align them to the first experimental peak, ${ }^{91}$ whose position we estimated to be at $285 \mathrm{eV}$. The applied shift is $-0.44 \mathrm{eV}$ for Dunning's set, and $-0.91 \mathrm{eV}$ for Pople's set. Inclusion of Rydberg-type functions in the basis set has a more modest effect than in the case of ethylene.

NTOs of the most intense core excitations obtained with the $6-311++\mathrm{G}^{* *}$ basis set are shown in Table 1, allowing us to identify from which of the two $\mathrm{C}$ atoms they originate from and the character of the transition. The Valence/Rydberg character of these intense core excitations is quantified in Table 2.

The X-ray absorption spectra obtained at the fluorine edge of $\mathrm{CH}_{2} \mathrm{CHF}$ are shown in Fig. 7; the raw data are given in Table S13. In the experimental spectrum, digitized from Ref. 91, only two peaks are clearly discernible, with absolute energies assigned at $(689.2 \pm 2.0) \mathrm{eV}$ and $(690.6 \pm 2.0) \mathrm{eV}\left(1 s_{\mathrm{F}} \rightarrow \sigma^{*}(\mathrm{C}-\mathrm{F})\right)$. In the experimental study, the first peak is assigned to a $1 s_{\mathrm{F}} \rightarrow \pi^{*}(\mathrm{C}=\mathrm{C})$ transition, and the second one to a $1 s_{\mathrm{F}} \rightarrow \sigma^{*}(\mathrm{C}-\mathrm{F})$ transition. Inspection of the results in Table S13 and of the NTOs in Table 3 indicates that the first band results from two almost degenerate transitions, $1 s_{\mathrm{F}} \rightarrow \sigma^{*}(\mathrm{C}-\mathrm{F})$ and $1 s_{\mathrm{F}} \rightarrow \pi^{*}(\mathrm{C}=\mathrm{C})$. The third excitation (second experimental band) also appears to be of $1 s_{\mathrm{F}} \rightarrow \sigma^{*}$ (C-F) character.

The experimental IE is at $693.26 \mathrm{eV} \cdot{ }^{97}$ The computed spectra in the Pople set (with and without Rydberg functions) are realigned by $-1.98 \mathrm{eV}$, and those for the Dunning basis by $-1.58 \mathrm{eV}$. 


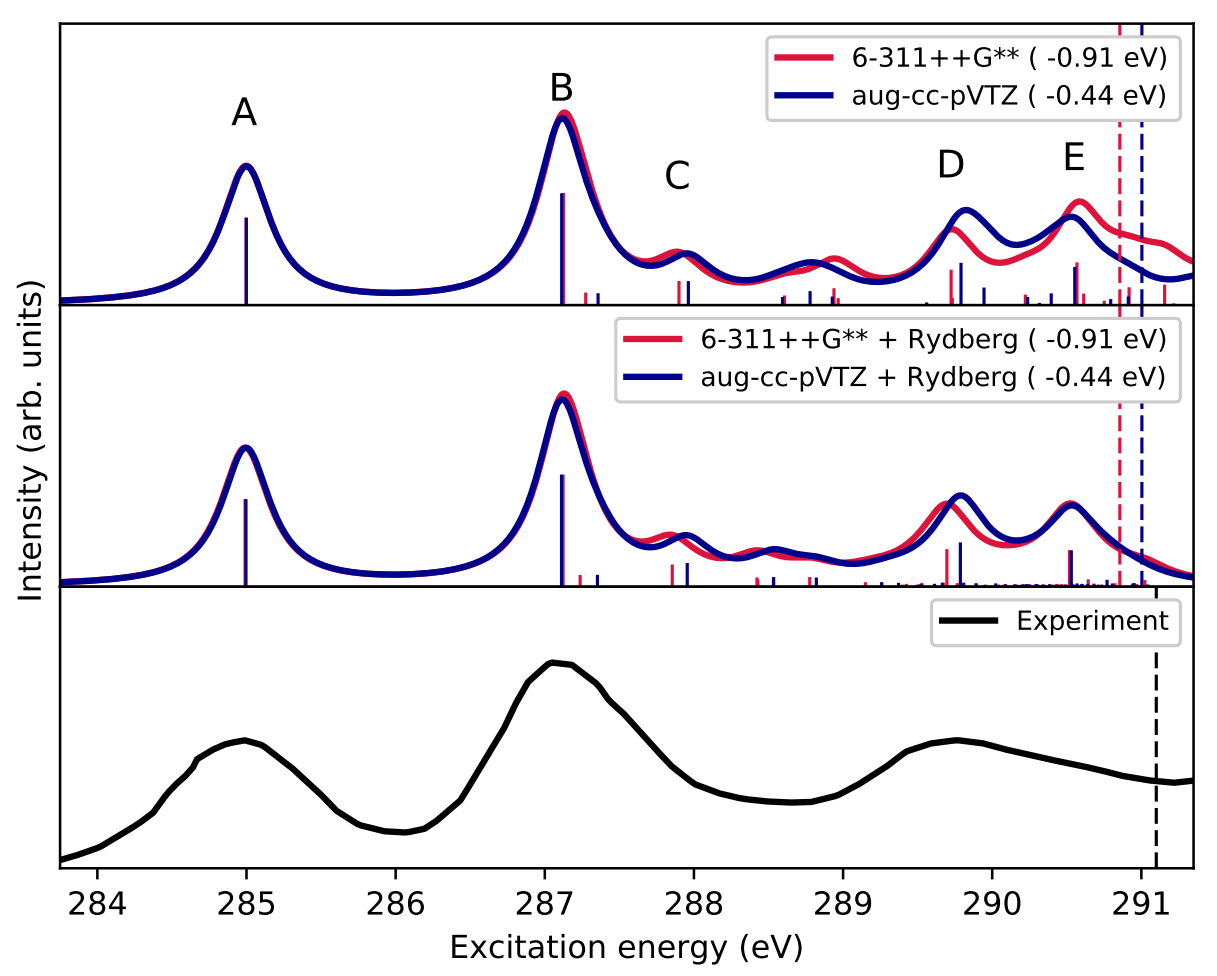

Figure 6: Vinyl fluoride. fc-CVS-EOMEE-CCSD X-ray absorption spectra at the C-edge obtained by convolution of the computed energies and oscillator strengths with a Lorentzian function $(\mathrm{FWHM}=0.4 \mathrm{eV})$. The experimental spectrum $(0.6 \mathrm{eV}$ resolution) was digitized from Ref. 91. The dashed vertical lines correspond to the IEs of the $1 s$ electron on the carbon atom of the $\mathrm{CH}_{2}$ group. The IEs of the $1 \mathrm{~s}$ electron of $\mathrm{C}_{\mathrm{CHF}}$ atom are outside the displayed frequency range (experimental IE $293.48 \mathrm{eV}$ ). The energy shifts required to align the NEXAFS profiles in each basis set with the experimental one are indicated in parenthesis. The computed IEs have been shifted by the same amount as used to align the NEXAFS profiles. 
Table 1: Vinylfluoride. fc-CVS-EOM-CCSD/6-311++G** NTOs of 5 selected core-excited states at the $\mathrm{C}$ edge (NTO isosurface is 0.05 ).

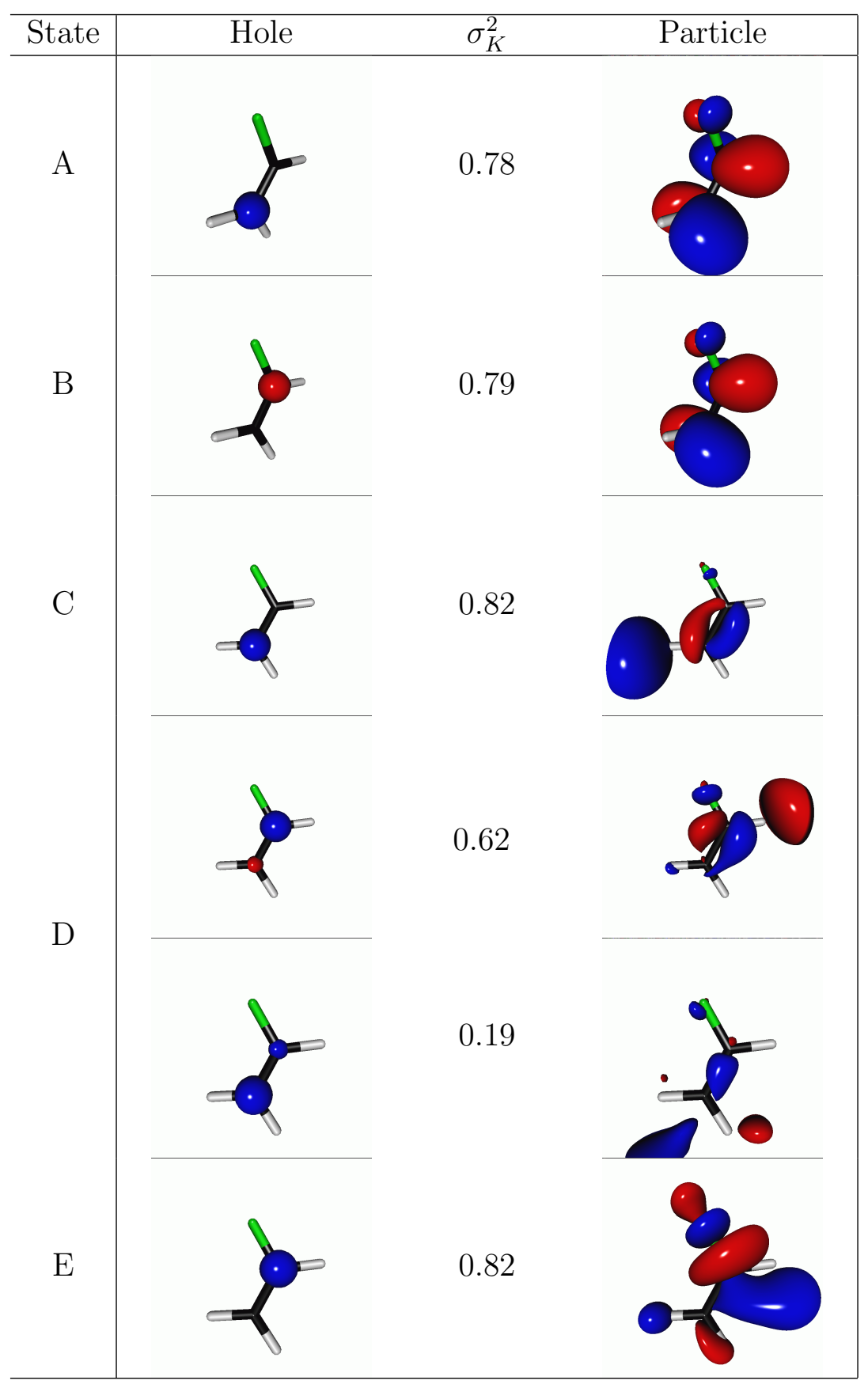


Table 2: Vinylfluoride. Changes in the second moments of charge density (in $\AA^{2}$ ) and electron and hole size components (in $\AA$ ) for selected core-excited states at the C K-edge.

\begin{tabular}{c|cccc|cccc|cccc}
\hline State & $\Delta\left\langle x^{2}\right\rangle$ & $\Delta\left\langle y^{2}\right\rangle$ & $\Delta\left\langle z^{2}\right\rangle$ & $\Delta\left\langle r^{2}\right\rangle$ & $x_{e}$ & $y_{e}$ & $z_{e}$ & $r_{e}$ & $x_{h}$ & $y_{h}$ & $z_{h}$ & $r_{h}$ \\
\hline $\mathrm{A}$ & -0.35 & -0.05 & 0.36 & -0.05 & 1.92 & 0.68 & 0.81 & 1.40 & 0.10 & 0.10 & 0.10 & 0.17 \\
$\mathrm{~B}$ & 0.36 & -0.17 & 0.38 & 0.57 & 0.92 & 0.67 & 0.81 & 1.39 & 0.10 & 0.10 & 0.10 & 0.17 \\
$\mathrm{C}$ & 1.57 & 5.80 & 1.11 & 8.49 & 1.42 & 2.39 & 1.22 & 3.03 & 0.10 & 0.10 & 0.10 & 0.17 \\
$\mathrm{D}$ & 2.16 & 3.86 & 1.50 & 7.52 & 1.81 & 1.78 & 1.36 & 2.88 & 0.51 & 0.29 & 0.10 & 0.59 \\
$\mathrm{E}$ & 1.24 & 2.43 & 0.73 & 4.40 & 1.50 & 1.58 & 1.03 & 2.40 & 0.10 & 0.10 & 0.10 & 0.17 \\
\hline
\end{tabular}

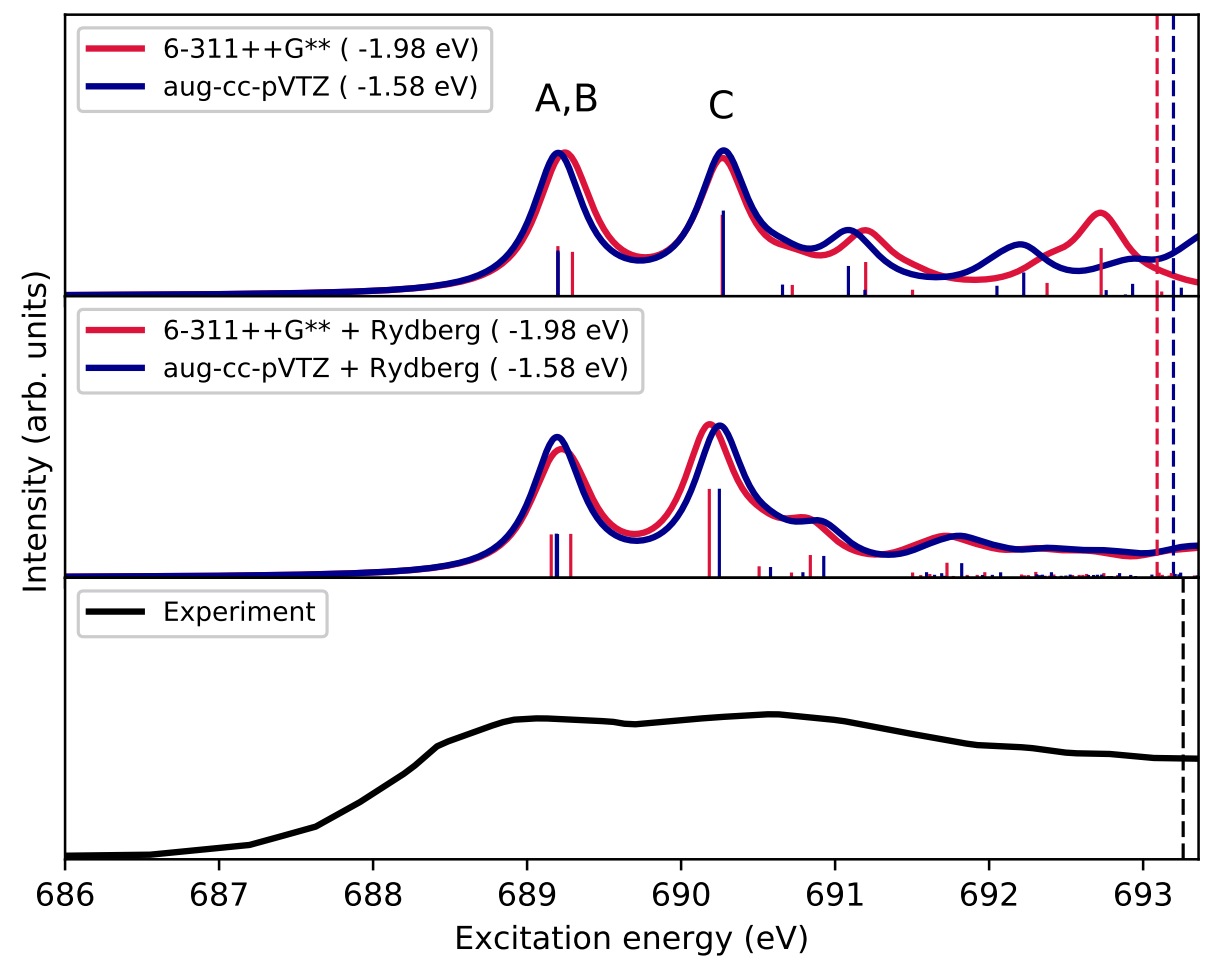

Figure 7: Vinylfluoride. fc-CVS-EOMEE-CCSD X-ray absorption spectra at the fluorine $\mathrm{K}$ edge, obtained by convolution of the computed energies and oscillator strengths with a Lorentzian function $(\mathrm{FWHM}=0.4 \mathrm{eV})$. The experimental spectrum was digitized from Ref. 91. The dashed vertical lines correspond to the IEs. The energy shifts required to align the NEXAFS profiles in each basis set with the experimental one are indicated in parenthesis. The computed IEs have been shifted by the same amount as used to align the NEXAFS profiles. The shift was computed based on the experimentally derived maximum at 689.2 eV. 
Table 3: Vinylfluoride. fc-CVS-EOMEE-CCSD/6-311++G** NTOs of 3 selected coreexcited states at the F edge. NTO isosurface is 0.05 .

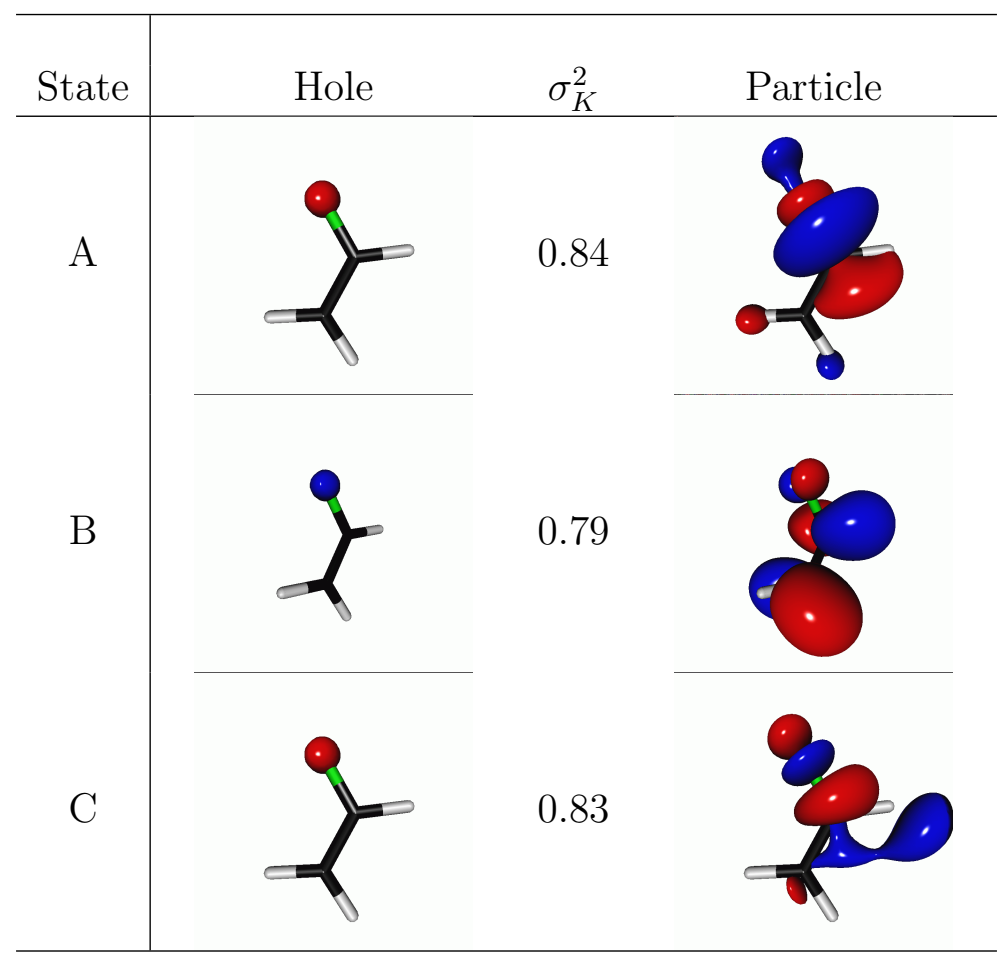


Fig. 8 shows the fc-CVS-EOMEE-CCSD NEXAFS spectra of $\mathrm{O}_{3}$, based on the spectral data in Table S14. This molecule displays the largest overall shift relative to the experimental spectrum, ${ }^{92}-2.35 \mathrm{eV}$ in the Pople set, and $-1.96 \mathrm{eV}$ with Dunning's set. Apart from this, our calculations confirm the assignment in Ref. 92: the first spectral feature is due to the terminal oxygens' $1 s \rightarrow \pi^{\star}$, whereas the second (broad) band is due to both the central oxygens $1 s \rightarrow \pi^{\star}$ and the terminal oxygens' $1 s \rightarrow \sigma^{\star}$ excitations, see also the NTOs in Table 4. The shoulder at $530.7 \mathrm{eV}$ in the experimental spectrum is known to be due to the $\mathrm{O} 1 s \rightarrow \sigma^{\star}$ transition of a small amount of $\mathrm{O}_{2}$ present in the sample. ${ }^{92}$

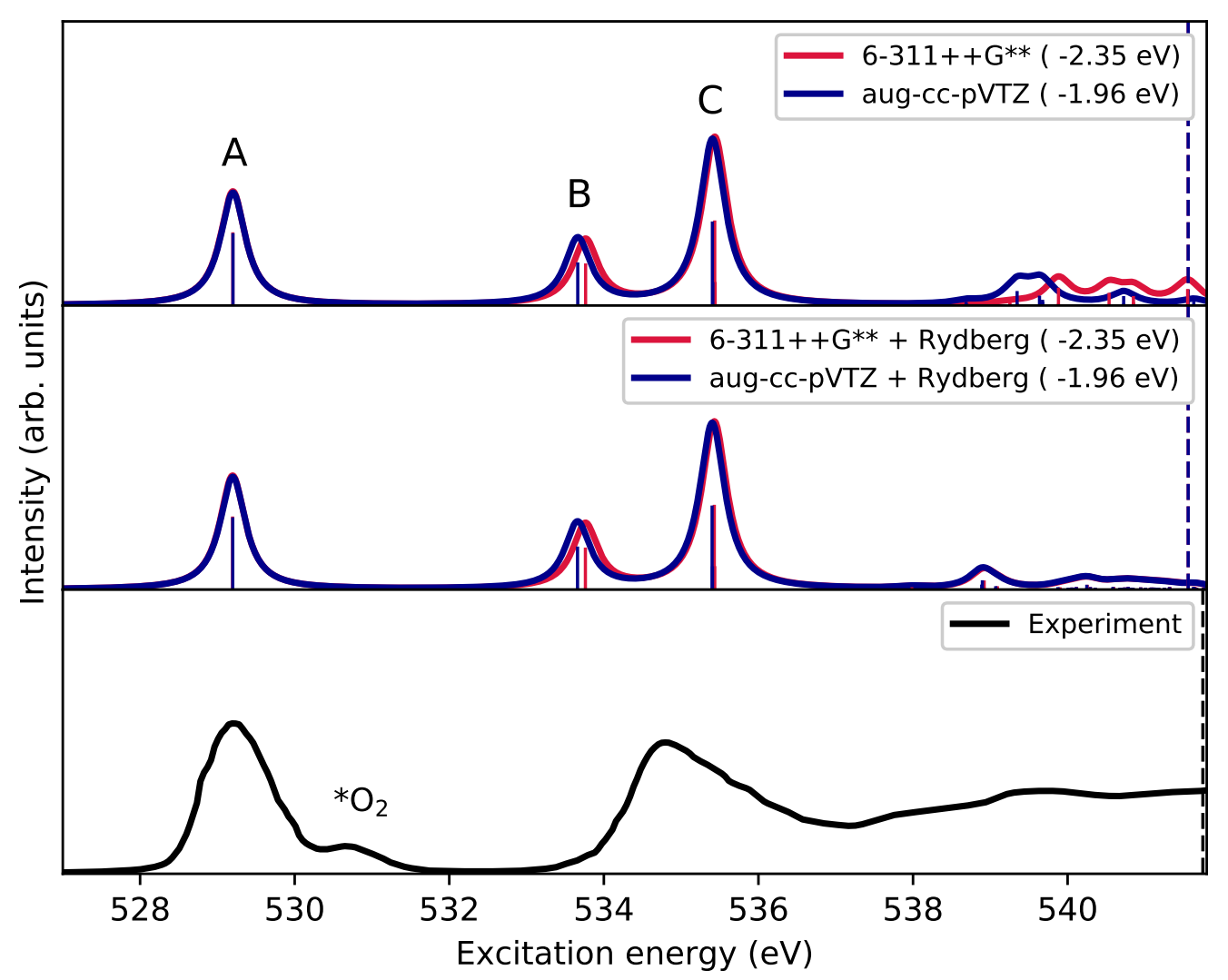

Figure 8: Ozone. fc-CVS-EOMEE-CCSD X-ray absorption spectra obtained from convolution with a Lorentzian function $(\mathrm{FWHM}=0.4 \mathrm{eV})$ of the computed excitation energies and oscillator strengths. The experimental spectrum High-Resolution Inner-Shell (0.05 eV resolution) was digitized from Ref. 92. The dashed vertical lines correspond to the ionization energy of the terminal $\mathrm{O}$ atom. The central oxygen's IE was omitted in figures as it lies above $545 \mathrm{eV}$. 
Table 4: Ozone. fc-CVS-EOMEE-CCSD/6-311++G $\mathrm{G}^{* *}$ NTOs of the first 3 core-excited states. NTO isosurface is 0.05

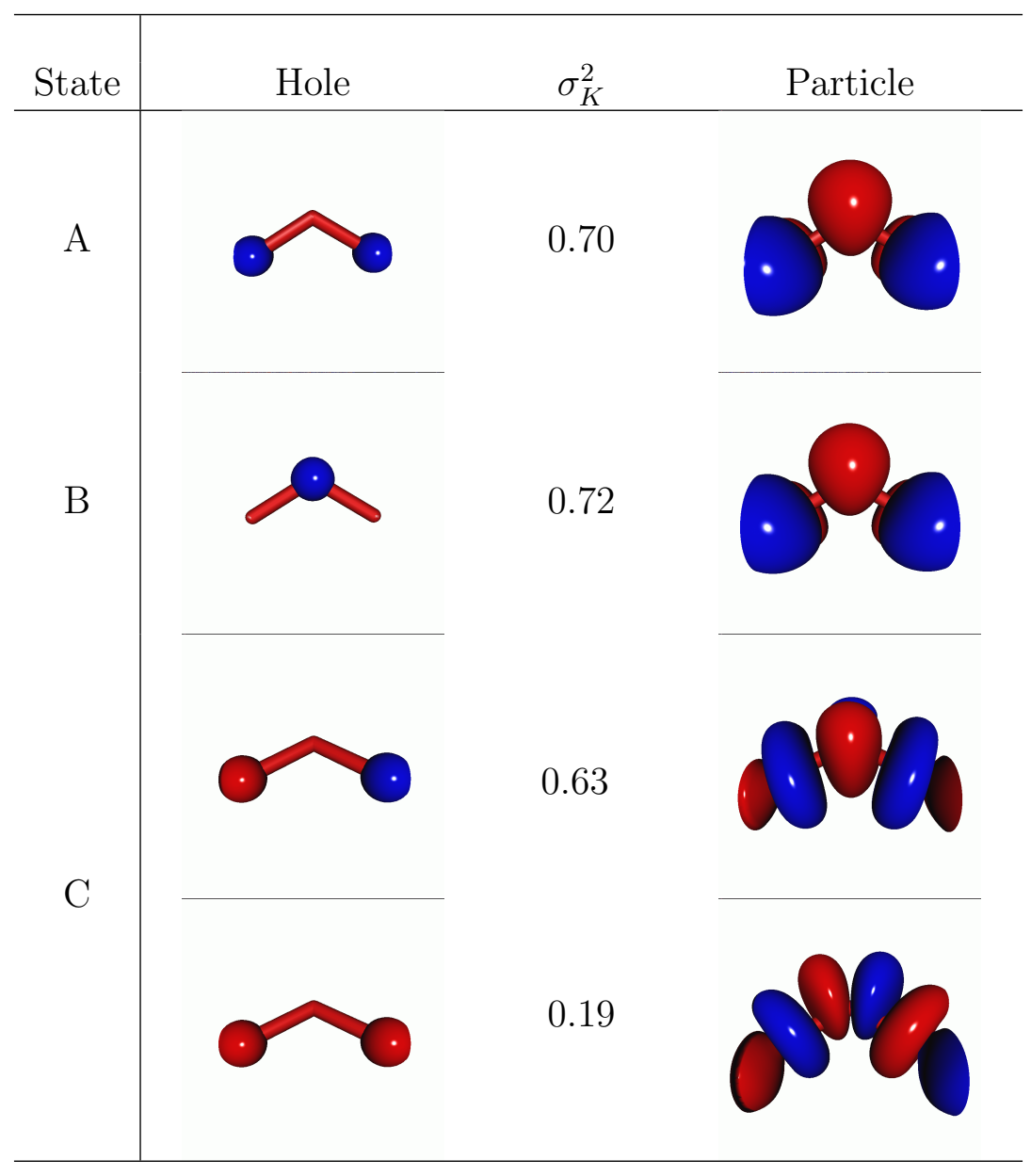

The final system considered here is adenine, whose NEXAFS and XPS spectra were experimentally recorded in gas-phase by Plekan et al. ${ }^{86}$ We considered both carbon and nitrogen edges. Due to the relatively large size of this system, we only carried out calculations in the $6-311++\mathrm{G}^{* *}$ basis set. The upper and lower panels of Figure 9 show the $\mathrm{C}$ and $\mathrm{N}$ K-edge spectra, respectively. The raw data are given in Table S15. The C K-edge spectra were shifted by -1.10 (non-planar)/-1.14(planar) eV, and the N K-edge one by -1.43(nonplanar)/-1.45(planar) eV, and one can expect an even smaller shift had the larger aug-ccpVTZ basis set been used. The experimental features are, once again, quite well reproduced. 
The agreement for the higher-energy peaks could probably be further improved by inclusion of Rydberg functions. Remarkably, the C-edge spectrum obtained from the planar geometry is more similar to the experimental spectrum, primarily due to the larger splitting between the 4th and 5th excitations in the non-planar structure. The spectral assignment for both structures is, nonetheless, identical. This is best appreciated looking at the NTOs for the first 5 excitations shown in Table 5. We also note that in this case, as in other examples, NTOs reveal that the electronic transitions have rather simple character and can be described by a single NTO pair. In contrast, the EOM wavefunctions often show multiple amplitudes with comparable weights, giving a misleading impression of the character of the transition. 

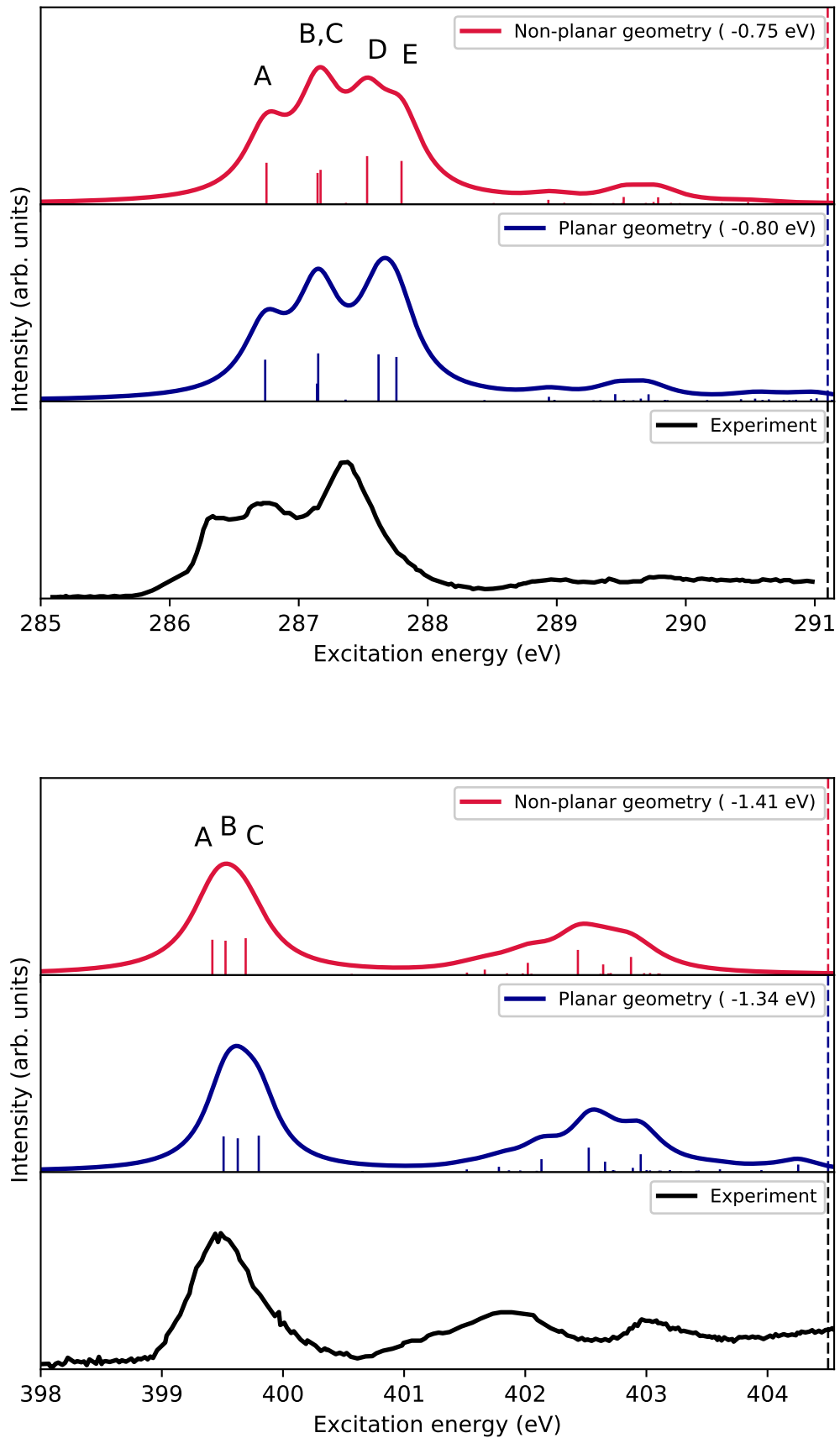

Figure 9: Adenine. C-edge (upper panels) and N-edge (lower panels) fc-CVS-EOMEECCSD/6-311++G** X-ray absorption spectra for two different molecular structures, obtained by convolution of the computed energies and oscillator strengths with a Lorentzian function $(\mathrm{FWHM}=0.4 \mathrm{eV})$. The rigid shifts applied are indicated in parenthesis in the legends. They were determined with respect to the first experimental peak position in each spectrum, estimated to be at $286.4 \mathrm{eV}$ for $\mathrm{C}$ and $399.4 \mathrm{eV}$ for $\mathrm{N}$. The vertical dashed line correspond to the first IE. The computed IEs have been shifted by the same amount as used to align the NEXAFS profiles. The experimental spectra $(0.57 \mathrm{eV}$ resolution at the $\mathrm{C}$ K-edge and $0.59 \mathrm{eV}$ resolution at the N K-edge) were digitized from Ref. 86. 
Table 5: Adenine. fc-CVS-EOMEE-CCSD/6-311++G** NTOs of the first 5 core-excited states at the $\mathrm{C}$ K-edge at the non-planar RI-MP2/cc-pVTZ geometry (left) and planar $\mathrm{B} 3 \mathrm{LYP} / \mathrm{cc}-\mathrm{pVTZ}$ geometry (right). NTO isosurface is 0.05 .

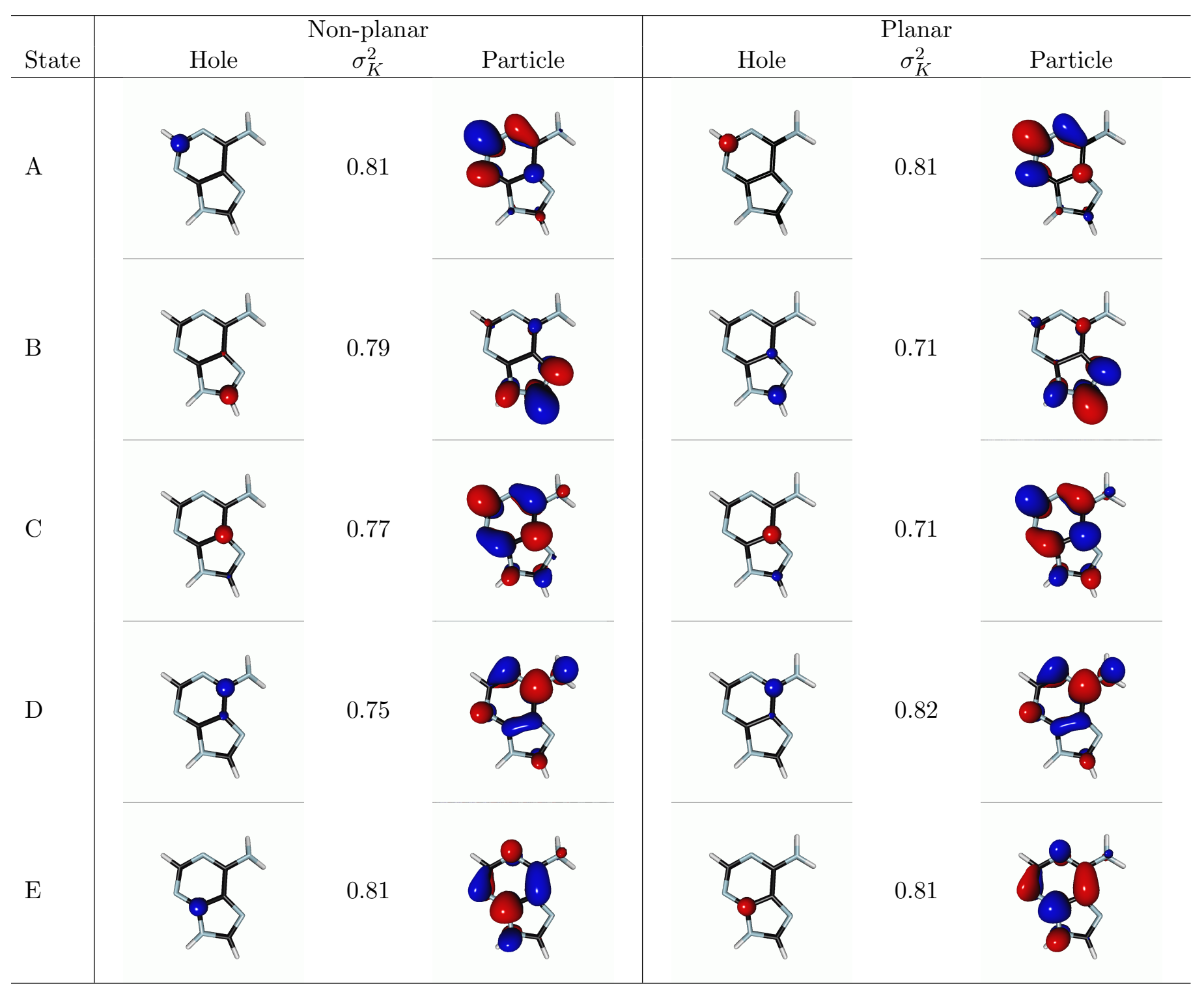


Table 6: Adenine. fc-CVS-EOMEE-CCSD/6-311++ $\mathrm{G}^{* *}$ NTOs of the first 3 core-excited states at the $\mathrm{N}$ K-edge at the non-planar RI-MP2/cc-pVTZ geometry (left) and planar B3LYP/cc-pVTZ geometry. NTO isosurface is 0.05.

State

\subsection{Core-level transient absorption spectroscopy}

The advances in X-ray Free-Electron Lasers in the last decade have boosted the interest in computational methodologies to simulate of Time-Resolved X-ray Absorption (TR-XAS or TR-NEXAFS). ${ }^{8,9,98,99}$ Typically, in TR-NEXAFS pump-probe experiments, the sample is first brought to a valence excited state by a UV pulse, and then probed, at different time delays, with X-ray radiation. To simulate these processes, methods to compute the intensity of valence-to-core transitions are needed. An EOM-CCSD/CC3 methodology, based on the CVS approach of Ref. 28, has been devised and used, for instance, to simulate and interpret TR-NEXAFS experiments in thymine. ${ }^{8}$ The study aimed at assessing the ability of K-edge resonant absorption spectroscopy to probe ultrafast $\pi \pi^{\star} / \mathrm{n} \pi^{\star}$ internal conversion in organic 
chromophores. Other methodologies have also been devised within the ADC, ${ }^{100-102}$ (MOM)TDDFT $^{99}$ and TP-DFT ${ }^{13}$ frameworks.

We have extended the fc-CVS-EOMEE-CCSD formalism to the computation of the transition density matrices between two excited states, from which the transient X-ray absorption spectra can then be obtained. As illustrative example, we have considered the valence-tocore spectra of uracil at the $\mathrm{O}, \mathrm{C}$ and $\mathrm{N}$ edges. TR-NEXAFS spectra of uracil have not been experimentally measured yet, but they are expected to bear strong similarities with those of thymine, whose O-edge TR-NEXAFS was measured in Ref. 8. Two valence excited states were considered, the first bright $\pi \pi^{*}$ state $\left(\mathrm{S}_{2}\right.$ at $\mathrm{FC}$ geometry) and the first dark $\mathrm{n}_{O} \pi^{*}$ $\left(\mathrm{S}_{1}\right.$ at $\mathrm{FC}$ geometry) state. The NTOs of these two states, obtained at the Franck-Condon geometry, are shown in Table 7.

Table 7: Uracil. EOMEE-CCSD/6-311++G** NTOs of the first 2 valence excited states and fc-CVS-EOMEE-CCSD/6-311++ $\mathrm{G}^{* *} \mathrm{NTO}$ of the core excitation from the $\mathrm{S}_{1}$ valence excited state. NTO isosurface is 0.05 .

Excitation

Given the localized nature of the $\mathrm{n}_{O} \pi^{*}\left(\mathrm{~S}_{1}\right)$ state on one of the two oxygen nuclei, and 


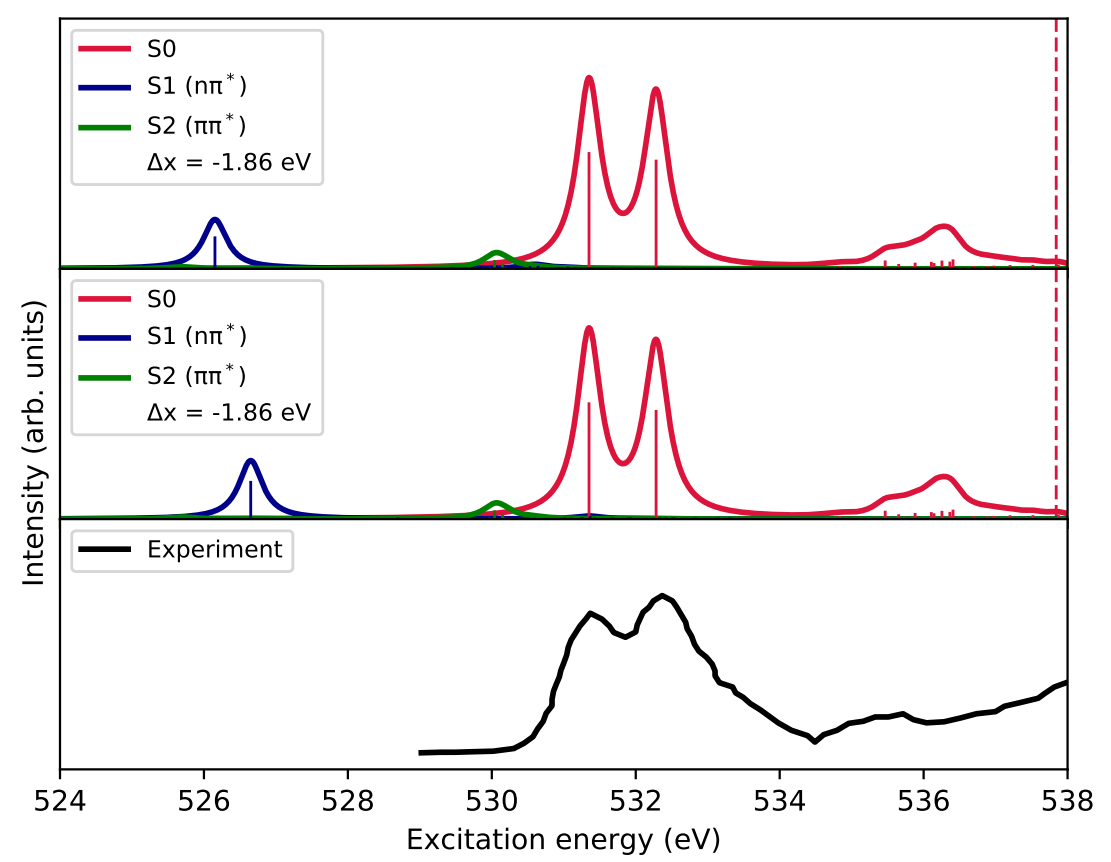

Figure 10: O-edge of uracil. Upper panel: fc-CVS-EOMEE-CCSD/6-311++G** ground and excited-state core absorption spectra at the DFT Franck-Condon geometry of Ref. 83. Lower panel: fc-CVS-EOMEE-CCSD/6-311++ $\mathrm{G}^{* *}$ ground and excited-state core-absorption spectra, at the Franck-Condon geometry for both the ground state $\left(\mathrm{S}_{0}\right)$ and the $\pi \pi^{*}\left(\mathrm{~S}_{2}\right)$ states, and at the TD-DFT optimized $\mathrm{S}_{1}$ geometry of Ref. 83. for $\mathrm{S}_{1}$. In both cases, a Lorentzian convolution function $(\mathrm{FWHM}=0.4 \mathrm{eV})$ was used. 


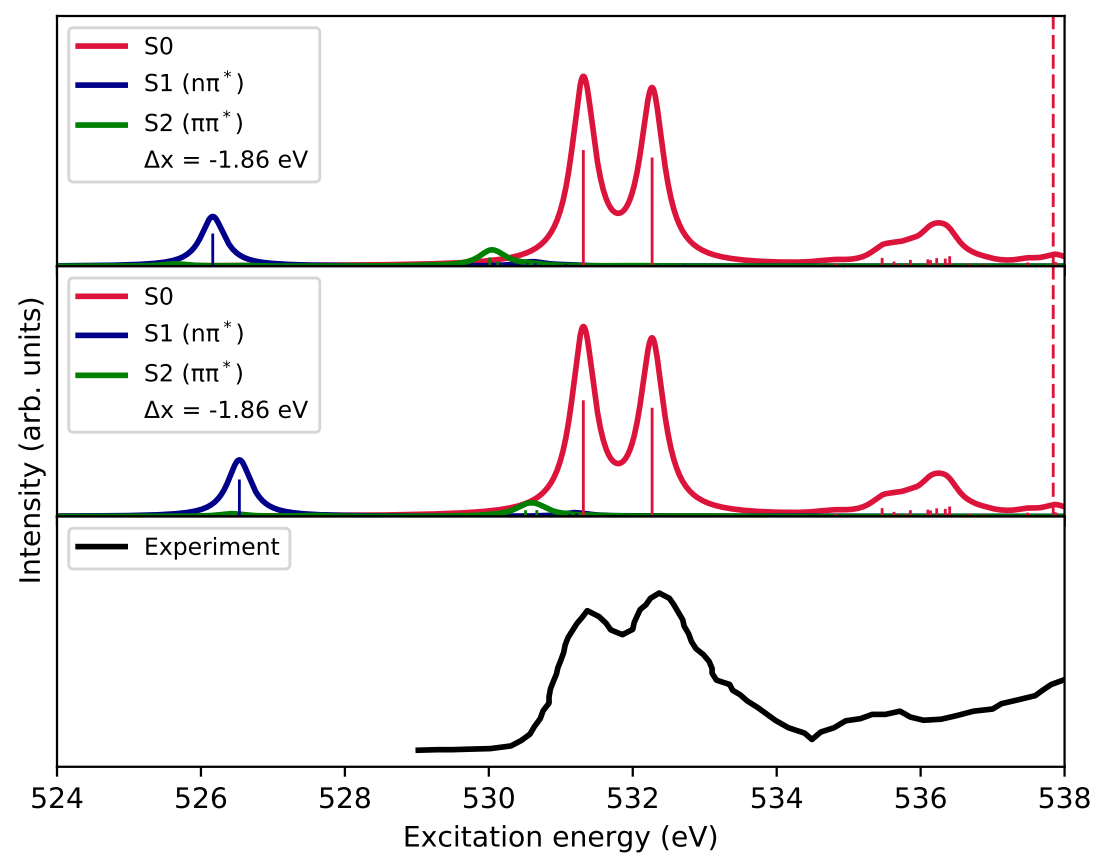

Figure 11: O-edge of uracil. Upper panel: fc-CVS-EOMEE-CCSD/6-311++G** ground and excited-state core-absorption spectra at the optimized MP2/cc-pVTZ Franck-Condon geometry. Lower panel: fc-CVS-EOMEE-CCSD/6-311++ $\mathrm{G}^{* *}$ ground and excited-state coreabsorption spectra at planar optimized geometries for each state, i.e., MP2/cc-pVTZ for the ground state, and EOM-CCSD/aug-cc-pVDZ for the two valence excited states. In both cases, a Lorentzian convolution function $(\mathrm{FWHM}=0.4 \mathrm{eV})$ was used.

To conclude this section, we have also considered the transient state spectra that one could expect to observe if probing at the $\mathrm{C}$ and $\mathrm{N}$ edges after the initial pump, along with the computed ground state NEXAFS spectra and their experimental counterparts. Fig. 12 shows that at the C-edge the valence-to-core spectra are rather weak (signals have been enhanced by a factor 10 in the figures), and that, opposite to the O-edge case, the most intense features at this edge originate from the $\pi \pi^{*}$ excited state. At the N-edge (see Fig. 13) the intensities of the transient absorption spectra are higher than at the $\mathrm{C}$ edge (signals have been enhanced by a factor 5 in the figures) and, as in the $\mathrm{C}$ edge case, the dominant features are from the $\pi \pi^{*}$ excited state. 

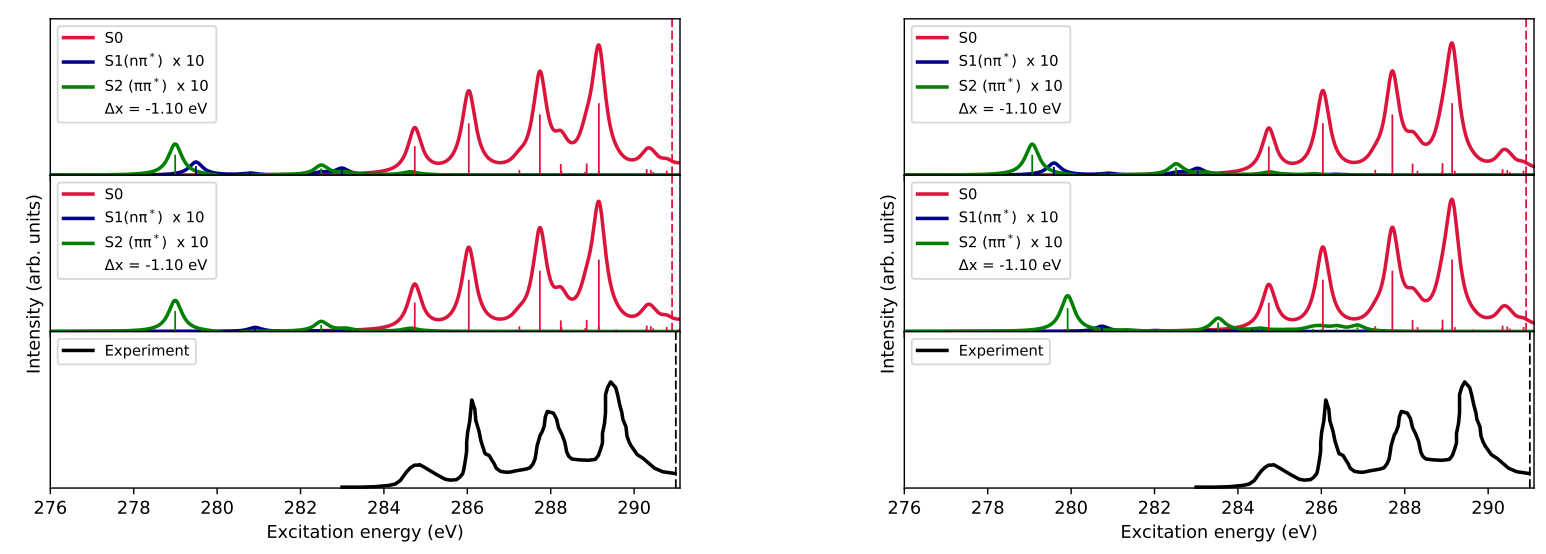

Figure 12: C-edge of uracil. fc-CVS-EOMEE-CCSD/6-311++G** ground and excited-state core-absorption spectra at different geometries. On the left, DFT geometries of Ref. 83; at the Franck-Condon DFT geometry for all states on the upper panel, and the FranckCondon DFT geometry for $\mathrm{S}_{0}$ and $\mathrm{S}_{2}$ and the TD-DFT optimized $S_{1}$ geometry for $\mathrm{S}_{1}$ on the middle panel. On the right, the planar MP2/cc-pVTZ Franck-Condon geometry for all states on the upper panel, and the MP2 optimized geometry for $\mathrm{S}_{0}$ and planar optimized EOM-CCSD/aug-cc-pVDZ for both $\mathrm{S}_{1}$ and $\mathrm{S}_{2}$ on the middle panel.

Figure 13: N-edge of uracil. fc-CVS-EOMEE-CCSD/6-311++G** ground and excited-state core-absorption spectra at different geometries: the Franck-Condon DFT geometry of Ref. 83 for all states on left upper panel; the Franck-Condon DFT geometry of Ref. 83 for $\mathrm{S}_{0}$ and $\mathrm{S}_{2}$ and the TD-DFT optimized $\mathrm{S}_{1}$ geometry for $\mathrm{S}_{1}$ on left lower panel; the planar MP2/cc-pVTZ Franck-Condon geometry for all states on right upper panel; the MP2 optimized geometry for $\mathrm{S}_{0}$ and planar optimized EOM-CCSD/aug-cc-pVDZ geometry for both $\mathrm{S}_{1}$ and $\mathrm{S}_{2}$ on the bottom right panel.
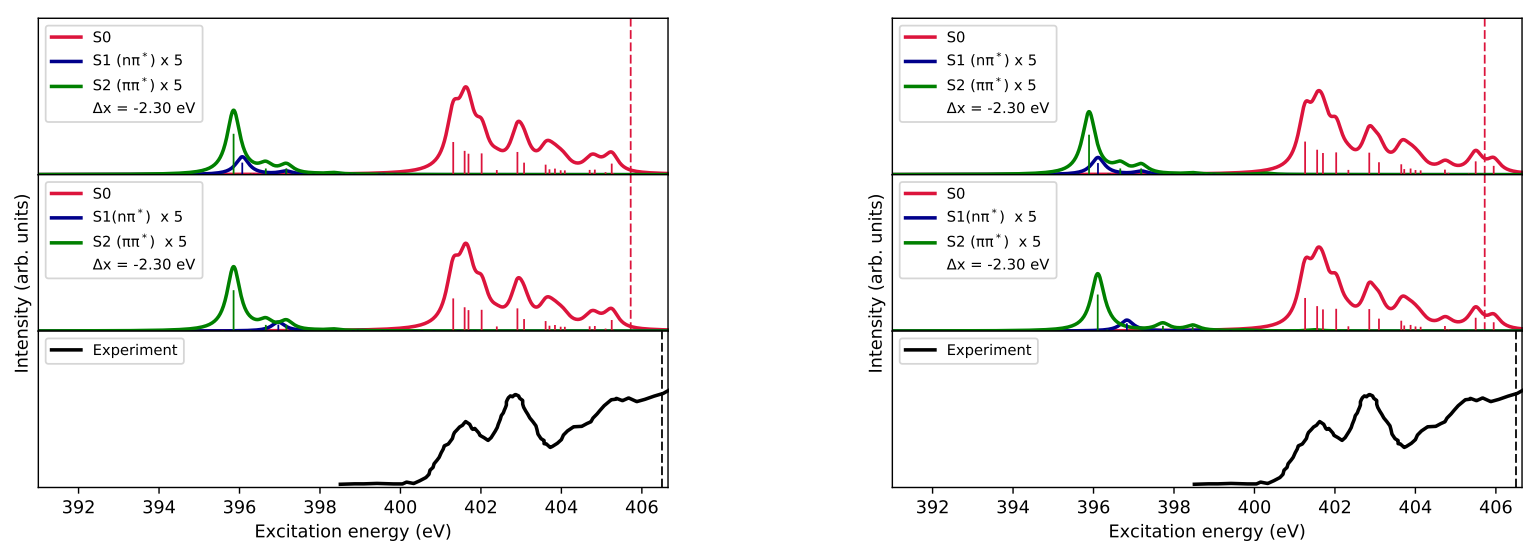


\section{Conclusions}

We have presented a new, fully-analytic core-valence separated equation-of-motion approach, named fc-CVS-EOM-CCSD, for calculating spectral descriptors of X-ray absorption spectroscopies, specifically near-edge absorption fine structure, core-ionization energies and transientstate (time-resolved) X-ray absorption. The approach exploits the large energy separation of the core and valence orbitals both in the determination of the amplitudes of both coupledcluster ground-state wavefunction (via the frozen-core condition) and of the EOM targetstate wavefunctions (via CVS). By decoupling core-level states from the high-lying valence states that are embedded in the ionization continuum, CVS addresses the most challenging issue in modeling core states, that is, complications due to their autoionizing nature. In contrast to full EOM-CCSD, fc-CVS-EOM-CCSD features a robust numeric performance and reduced computational costs. Yet, it retains sufficient correlation, necessary to describe orbital relaxation effects, and is size intensive by construction.

We benchmarked the method using a number of atomic and molecular systems, comparing the results with both a previously proposed CVS-CCSD scheme ${ }^{28}$ and with experimental data. The shape of computed NEXAFS spectra agrees very well with the experimental one in terms of the relative heights of the individual peaks and the distance between them. However, the computed spectra are shifted with respect to the experiment. The magnitude of the shifts required for the alignment varies between $0.2 \mathrm{eV}$ and $3 \mathrm{eV}$, depending on the edge and basis set considered. In all cases, the shifts are smaller than those obtained with the previously presented CVS-CCSD approach based on the energy separation between core and valence excited states, ${ }^{28}$ whereas the spectral profiles are essentially the same. Importantly, for all examples, we observed a systematic decrease of the shift upon a basis set increase.

The reduced absolute shifts from experiments compared to the previously presented CVS-CCSD approach ${ }^{28}$ are most likely due to effective error cancellation between the neglect of core correlation in the ground state, its inclusion in the excited states and the neglect of higher-order correlation and excitation effects in the (EOM-)CCSD method. Even if one 


\section{Acknowledgement}

M.L.V. and S.C. acknowledge support from DTU Chemistry (start-up Ph.D. grant). S.C. acknowledges support from the Independent Research Fund Denmark - DFF-Forskningsprojekt2 grant no. 7014-00258B and from the H2020-MSCA-ITN-2017 training network "COSINE - COmputational Spectroscopy In Natural sciences and Engineering". A.I.K. acknowledges support by the U.S. National Science Foundation (No. CHE-1566428) and by the Simons Foundation.

Note: The authors declare the following competing financial interest(s): A.I.K. is a part owner and a board member of Q-Chem, Inc.

Supporting Information Available: Tables with raw spectral data, proof of size extensivity, comparison with CVS-LRCCSD results and implementation formulas. This information is available free of charge via the Internet at http://pubs.acs.org. 


\section{References}

(1) van Bokhoven, J., Lamberti, C., Eds. X-Ray Absorption and X-ray Emission Spectroscopy; Theory and Applications; Wiley \& Sons, 2016.

(2) Mobilio, S., Boscherini, F., Meneghini, C., Eds. Synchrotron Radiation: Basics, Methods and Applications; Springer, 2014.

(3) Bergmann, U., Yachandra, V., Yano, J., Eds. X-Ray Free Electron Lasers: Applications in Materials, Chemistry and Biology; Energy and Environment Series 18; Royal Society of Chemistry, 2017.

(4) Nisoli, M.; Decleva, P.; Calegari, F.; Palacios, A.; Martín, F. Attosecond Electron Dynamics in Molecules. Chem. Rev. 2017, 117, 10760-10825.

(5) Norman, P.; Dreuw, A. Simulating X-ray Spectroscopies and Calculating Core-Excited States of Molecules. Chem. Rev. 2018, 118, 7208-7248.

(6) Stöhr, J. NEXAFS Spectroscopy; Springer, Berlin, 1992.

(7) Kostko, O.; Bandyopadhyay, B.; Ahmed, M. Vacuum Ultraviolet Photoionization of Complex Chemical Systems. Annu. Rev. Phys. Chem. 2016, 67, 19-40.

(8) Wolf, T.; Myhre, R.; Cryan, J.; Coriani, S.; Squibb, R.; Battistoni, A.; Berrah, N.; Bostedt, C.; Bucksbaum, P.; Coslovich, G.; Feifel, R.; Gaffney, K.; Grilj, J.; Martinez, T.; Miyabe, S.; Moeller, S.; Mucke, M.; Natan, A.; Obaid, R.; Osipov, T.; Plekan, O.; Wang, S.; Koch, H.; Gühr, M. Probing ultrafast $\pi \pi^{*} / \mathrm{n} \pi^{*}$ internal conversion in organic chromophores via K-edge resonant absorption. Nature Commun. 2017, $8,1-7$.

(9) Milne, C.; Penfold, T.; Chergui, M. Recent experimental and theoretical developments in time-resolved X-ray spectroscopies. Coord. Chem. Rev. 2014, 277-278, 44-68. 
(10) Kraus, P. M.; Zürch, M.; Cushing, S. K.; Neumark, D. M.; Leone, S. R. The ultrafast X- ray spectroscopic revolution in chemical dynamics. Nature Reviews Chemistry 2018, 2, 82-94.

(11) Besley, N.; Asmuruf, F. Time-dependent density functional theory calculations of the spectroscopy of core electrons. Phys. Chem. Chem. Phys. 2010, 12, 12024-12039.

(12) Triguero, L.; Pettersson, L. G. M.; Ågren, H. Calculations of near-edge x-rayabsorption spectra of gas-phase and chemisorbed molecules by means of densityfunctional and transition-potential theory. Phys. Rev. B 1998, 58, 8097-8110.

(13) Ehlert, C.; Gühr, M.; Saalfrank, P. An efficient first principles method for molecular pump-probe NEXAFS spectra: Application to thymine and azobenzene. J. Chem. Phys. 2018, 149, 144112.

(14) Evangelista, F. A.; Shushkov, P.; Tully, J. C. Orthogonality Constrained Density Functional Theory for Electronic Excited States. J. Phys. Chem. A 2013, 117, 7378-7392.

(15) Derricotte, W. D.; Evangelista, F. A. Simulation of X-ray absorption spectra with orthogonality constrained density functional theory. Phys. Chem. Chem. Phys. 2015, $17,14360-14374$.

(16) Helgaker, T.; Jørgensen, P.; Olsen, J. Molecular electronic structure theory; Wiley \& Sons, 2000.

(17) Koch, H.; Jørgensen, P. Coupled Cluster Response Functions. J. Chem. Phys. 1990, 93, 3333-3344.

(18) Stanton, J.; Bartlett, R. The equation of motion coupled-cluster method. A systematic biorthogonal approach to molecular excitation energies, transition probabilities, and excited state properties. J. Chem. Phys. 1993, 98, 7029-7039. 
(19) Christiansen, O.; Jørgensen, P.; Hättig, C. Response Functions from Fourier Component Variational Perturbation Theory Applied to a Time-Averaged Quasienergy. Int. J. Quantum Chem. 1998, 98, 1.

(20) Krylov, A. I. Equation-of-Motion Coupled-Cluster Methods for Open-Shell and Electronically Excited Species: The Hitchhiker's Guide to Fock Space. Ann. Rev. Phys. Chem. 2008, 59, 433-462.

(21) Helgaker, T.; Coriani, S.; Jørgensen, P.; Kristensen, K.; Olsen, J.; Ruud, K. Recent Advances in Wave Function-Based Methods of Molecular-Property Calculations. Chem. Rev. 2012, 112, 543-631.

(22) Bartlett, R. J. Coupled-cluster theory and its equation-of-motion extensions. WIREs Comput Mol Sci 2, 126-138.

(23) Christiansen, O. Coupled Cluster Theory with Emphasis on Selected New Developments. Theor. Chem. Acc. 2006, 116, 106.

(24) Sneskov, K.; Christiansen, O. Excited state coupled cluster methods. WIREs Comput. Mol. Sci. 2012, 2, 566-584.

(25) Coriani, S.; Fransson, T.; Christiansen, O.; Norman, P. Asymmetric-Lanczos-ChainDriven Implementation of Electronic Resonance Convergent Coupled-Cluster Linear Response Theory. J. Chem. Theory Comput. 2012, 8, 1616.

(26) Peng, B.; Lestrange, P. J.; Goings, J. J.; Caricato, M.; Li, X. Energy-Specific Equationof-Motion Coupled-Cluster Methods for High-Energy Excited States: Application to K-Edge X-Ray Absorption Spectroscopy. J. Chem. Theory Comput. 2015, 11, 4146.

(27) Coriani, S.; Christiansen, O.; Fransson, T.; Norman, P. Coupled-Cluster Response Theory for Near-Edge X-Ray-Absorption Fine Structure of Atoms and Molecules. Phys. Rev. A 2012, 85, 022507. 
(28) Coriani, S.; Koch, H. Communication: X-ray Absorption Spectra and Core-Ionization Potentials within a Core-Valence Separated Coupled Cluster Framework. J. Chem. Phys. 2015, 143, 181103.

(29) Krylov, A. I. In Reviews in Comp. Chem.; Parrill, A., Lipkowitz, K., Eds.; J. Wiley \& Sons, 2017; Vol. 30; Chapter 4, pp 151-224.

(30) Sadybekov, A.; Krylov, A. I. Coupled-cluster based approach for core-ionized and core-excited states in condensed phase: Theory and application to different protonated forms of aqueous glycine. J. Chem. Phys. 2017, 147, 014107.

(31) Hršak, D.; Nørby, M. S.; Coriani, S.; Kongsted, J. One-Photon Absorption Properties from a Hybrid Polarizable Density Embedding/Complex Polarization Propagator Approach for Polarizable Solutions. J. Chem. Theory Comput. 2018, 14, 2145-2154.

(32) Reinhardt, W. P. Complex coordinates in the theory of atomic and molecular structure and dynamics. Annu. Rev. Phys. Chem. 1982, 33, 223-255.

(33) Jagau, T.-C.; Bravaya, K. B.; Krylov, A. I. Extending Quantum Chemistry of Bound States to Electronic Resonances. Annu. Rev. Phys. Chem. 2017, 68, 525-553.

(34) Emrich, K. An extension of the coupled-cluster formalism to excited states (I). Nucl. Phys. 1981, A351, 379-396.

(35) Gilbert, A.; Besley, N.; Gill, P. Self-consistent field calculations of excited states using the maximum overlap method (MOM). J. Phys. Chem. A 2008, 112, 13164-13171.

(36) Zuev, D.; Vecharynski, E.; Yang, C.; Orms, N.; Krylov, A. I. New algorithms for iterative matrix-free eigensolvers in quantum chemistry. J. Comput. Chem. 2015, 36, $273-284$.

(37) Zuev, D.; Bravaya, K. B.; Crawford, T. D.; Lindh, R.; Krylov, A. I. Electronic structure 
of the two isomers of the anionic form of p-coumaric acid chromophore. J. Chem. Phys. 2011, 134, 034310 .

(38) Moiseyev, N. Non-Hermitian quantum mechanics; Cambridge University Press, 2011.

(39) Cederbaum, L. S.; Domcke, W.; Schirmer, J. Many-body theory of core holes. Phys. Rev. A: At. Mol. Opt. Phys. 1980, 22, 206-222.

(40) Barth, A.; Schirmer, J. Theoretical core-level excitation spectra of $\mathrm{N}_{2}$ and CO by a new polarisation propagator method. J. Phys. B: At. Mol. Phys. 1985, 18, 867-885.

(41) Wenzel, J.; Wormit, M.; Dreuw, A. Calculating Core-Level Excitations and X-Ray Absorption Spectra of Medium-Sized Closed-Shell Molecules with the AlgebraicDiagrammatic Construction Scheme for the Polarization Propagator. J. Comput. Chem. 2014, 35, 1900.

(42) Wenzel, J.; Holzer, A.; Wormit, M.; Dreuw, A. Analysis and Comparison of CVS-ADC Approaches up to Third Order for the Calculation of Core-Excited States. J. Chem. Phys. 2015, 142, 214104.

(43) Feshbach, H. A unified theory of nuclear reactions. 2. Ann. Phys. (N.Y.) 1962, 19, $287-313$.

(44) Fano, U. Configuration interaction on intensities and phase shifts. Phys. Rev. 1961, $124,1866-1878$.

(45) Fransson, T.; Coriani, S.; Christiansen, O.; Norman, P. Carbon X-ray Absorption Spectra of Fluoroethenes and Acetone: A Study at the Coupled Cluster, Density Functional, and Static-Exchange levels of Theory. J. Chem. Phys. 2013, 138, 124311.

(46) Löwdin, P.-O. Studies in perturbation theory. IV. Solution of eigenvalue problem by projection operator formalism. J. Math. Phys. 1962, 3, 969-982. 
(47) Averbukh, V.; Cederbaum, L. Ab initio calculation of interatomic decay rates by a combination of the Fano ansatz, Green's-function methods, and the Stieltjes imaging technique. J. Chem. Phys. 2005, 123, 204107.

(48) Sekino, H.; Bartlett, R. J. A linear response, coupled-cluster theory for excitation energy. Int. J. Quant. Chem. 1984, 26, 255-265.

(49) We use the standard convention that $i, j, k, l, \ldots$ refer to occupied orbitals, $a, b, c, d, \ldots$ represent virtual ones, while $p, q, r, s, \ldots$ denote generic orbitals, either occupied or unoccupied.

(50) Stanton, J.; Gauss, J. Analytic energy derivatives for ionized states described by the equation-of-motion coupled cluster method. J. Chem. Phys. 1994, 101, 8938-8944.

(51) Stanton, J.; Gauss, J. A discussion on some problems associated with the quantum mechanical treatment of open-shell molecules. Adv. Chem. Phys. 2003, 125, 101-146.

(52) Pieniazek, P. A.; Bradforth, S. E.; Krylov, A. I. Charge localization and Jahn-Teller distortions in the benzene dimer cation. J. Chem. Phys. 2008, 129, 074104.

(53) Löwdin, P.-O. Some Aspects on the Hamiltonian and Liouvillian Formalism, the Special Propagator Methods, and the Equation of Motion Approach. Adv. Quantum Chem. 1985, 17, 285-334.

(54) Levchenko, S. V.; Krylov, A. I. Equation-of-motion spin-flip coupled-cluster model with single and double substitutions: Theory and application to cyclobutadiene. J. Chem. Phys. 2004, 120, 175-185.

(55) Davidson, E. R. The iterative calculation of a few of the lowest eigenvalues and corresponding eigenvectors of large real-symmetric matrices. J. Comput. Phys. 1975, 17, $87-94$. 
(56) Hirao, K.; Nakatsuji, H. A generalization of the Davidson's method to large nonsymmetric eigenvalue problems. J. Comput. Phys. 1982, 45, 246-254.

(57) Rettrup, S. An iterative method for calculating several of the extreme eigensolutions of large real non-symmetric matrices. J. Comput. Phys. 1982, 45, 100-107.

(58) Stanton, J. F. Many-body methods for excited state potential energy surfaces. I. General theory of energy gradients for the equation-of-motion coupled-cluster method. $J$. Chem. Phys. 1993, 99, 8840-8847.

(59) Stanton, J. F.; Gauss, J. Analytic energy gradients for the equation-of-motion coupledcluster method: Implementation and application to the $\mathrm{HCN} / \mathrm{HNC}$ system. J. Chem. Phys. 1994, 100, 4695-4698.

(60) Levchenko, S. V.; Wang, T.; Krylov, A. I. Analytic gradients for the spin-conserving and spin-flipping equation-of-motion coupled-cluster models with single and double substitutions. J. Chem. Phys. 2005, 122, 224106.

(61) Nanda, K.; Krylov, A. I.; Gauss, J. Communication: The pole structure of the dynamical polarizability tensor in equation-of-motion coupled-cluster theory. J. Chem. Phys. 2018, 149, 141101.

(62) Luzanov, A.; Sukhorukov, A.; Umanskii, V. Application of transition density matrix for analysis of excited states. Theor. Exp. Chem. 1976, 10, 354-361.

(63) Luzanov, A.; Zhikol, O. In Practical aspects of computational chemistry I: An overview of the last two decades and current trends; Leszczynski, J., Shukla, M., Eds.; Springer, 2012 ; pp 415-449.

(64) Plasser, F.; Lischka, H. Analysis of excitonic and charge transfer interactions from quantum chemical calculations. J. Chem. Theory Comput. 2012, 8, 2777-2789. 

2

(65) Plasser, F.; Wormit, M.; Dreuw, A. New tools for the systematic analysis and visualization of electronic excitations. I. Formalism. J. Chem. Phys. 2014, 141, 024106-13.

(66) Plasser, F.; Bäppler, S.; Wormit, M.; Dreuw, A. New tools for the systematic analysis and visualization of electronic excitations. II. Applications. J. Chem. Phys. 2014, 141, 024107-12.

(67) Martin, R. Natural transition orbitals. J. Phys. Chem. A 2003, 118, 4775-4777.

(68) Bäppler, S.; Plasser, F.; Wormit, M.; Dreuw, A. Exciton analysis of many-body wave functions: Bridging the gap between the quasiparticle and molecular orbital pictures. Phys. Rev. A 2014, 90, 052521.

(69) Mewes, S.; Plasser, F.; Krylov, A. I.; Dreuw, A. Benchmarking excited-state calculations using exciton properties. J. Chem. Theory Comput. 2014, 11, 710-725.

(70) Schirmer, J. Beyond the random-phase approximation: A new approximation scheme for the polarization propagator. Phys. Rev. A 1982, 26, 2395-2416.

(71) Dreuw, A.; Wormit, M. The algebraic diagrammatic construction scheme for the polarization propagator for the calculation of excited states. WIREs Comput. Mol. Sci. 2015, 5, 82-95.

(72) Angonoa, G.; Walter, O.; Schirmer, J. Theoretical K-shell ionization spectra of N2 and CO by a fourth-order Greens function method. J. Chem. Phys. 1987, 87, 6789-6801.

(73) Schirmer, J.; Thiel, A. An intermediate state representation approach to K-shell ionization in molecules. I. Theory. J. Chem. Phys. 2001, 115, 10621.

(74) Purvis, G.; Bartlett, R. A full coupled-cluster singles and doubles model: the inclusion of disconnected triples. J. Chem. Phys. 1982, 76, 1910-1918. 
(75) Stanton, J.; Gauss, J. Perturbative treatment of the similarity transformed Hamiltonian in equation-of-motion coupled-cluster approximations. J. Chem. Phys. 1995, 103, 1064-1076.

(76) Wang, Z.; Tu, Z.; Wang, F. Equation-of-Motion Coupled-Cluster Theory for Excitation Energies of Closed-Shell Systems with Spin-Orbit Coupling. J. Chem. Theory Comput. 2014, 10, 5567-5576.

(77) Krylov, A. I.; Gill, P. M. W. Q-Chem: An engine for innovation. WIREs Comput. Mol. Sci. 2013, 3, 317-326.

(78) Shao, Y.; Gan, Z.; Epifanovsky, E.; Gilbert, A. T.; Wormit, M.; Kussmann, J.; Lange, A. W.; Behn, A.; Deng, J.; Feng, X.; Ghosh, D.; Goldey, M.; Horn, P. R.; Jacobson, L. D.; Kaliman, I.; Khaliullin, R. Z.; Kuś, T.; Landau, A.; Liu, J.; Proynov, E. I.; Rhee, Y. M.; Richard, R. M.; Rohrdanz, M. A.; Steele, R. P.; Sundstrom, E. J.; III, H. L. W.; Zimmerman, P. M.; Zuev, D.; Albrecht, B.; Alguire, E.; Austin, B.; Beran, G. J. O.; Bernard, Y. A.; Berquist, E.; Brandhorst, K.; Bravaya, K. B.; Brown, S. T.; Casanova, D.; Chang, C.-M.; Chen, Y.; Chien, S. H.; Closser, K. D.; Crittenden, D. L.; Diedenhofen, M.; Jr., R. A. D.; Do, H.; Dutoi, A. D.; Edgar, R. G.; Fatehi, S.; Fusti-Molnar, L.; Ghysels, A.; Golubeva-Zadorozhnaya, A.; Gomes, J.; HansonHeine, M. W.; Harbach, P. H.; Hauser, A. W.; Hohenstein, E. G.; Holden, Z. C.; Jagau, T.-C.; Ji, H.; Kaduk, B.; Khistyaev, K.; Kim, J.; Kim, J.; King, R. A.; Klunzinger, P.; Kosenkov, D.; Kowalczyk, T.; Krauter, C. M.; Lao, K. U.; Laurent, A. D.; Lawler, K. V.; Levchenko, S. V.; Lin, C. Y.; Liu, F.; Livshits, E.; Lochan, R. C.; Luenser, A.; Manohar, P.; Manzer, S. F.; Mao, S.-P.; Mardirossian, N.; Marenich, A. V.; Maurer, S. A.; Mayhall, N. J.; Neuscamman, E.; Oana, C. M.; Olivares-Amaya, R.; O’Neill, D. P.; Parkhill, J. A.; Perrine, T. M.; Peverati, R.; Prociuk, A.; Rehn, D. R.; Rosta, E.; Russ, N. J.; Sharada, S. M.; Sharma, S.; Small, D. W.; Sodt, A.; Stein, T.; Stck, D.; Su, Y.-C.; Thom, A. J.; Tsuchimochi, T.; Vanovschi, V.; Vogt, L.; Vy- 
drov, O.; Wang, T.; Watson, M. A.; Wenzel, J.; White, A.; Williams, C. F.; Yang, J.; Yeganeh, S.; Yost, S. R.; You, Z.-Q.; Zhang, I. Y.; Zhang, X.; Zhao, Y.; Brooks, B. R.; Chan, G. K.; Chipman, D. M.; Cramer, C. J.; III, W. A. G.; Gordon, M. S.; Hehre, W. J.; Klamt, A.; III, H. F. S.; Schmidt, M. W.; Sherrill, C. D.; Truhlar, D. G.; Warshel, A.; Xu, X.; Aspuru-Guzik, A.; Baer, R.; Bell, A. T.; Besley, N. A.; Chai, J.-D.; Dreuw, A.; Dunietz, B. D.; Furlani, T. R.; Gwaltney, S. R.; Hsu, C.-P.; Jung, Y.; Kong, J.; Lambrecht, D. S.; Liang, W.; Ochsenfeld, C.; Rassolov, V. A.; Slipchenko, L. V.; Subotnik, J. E.; Voorhis, T. V.; Herbert, J. M.; Krylov, A. I.; Gill, P. M.; Head-Gordon, M. Advances in molecular quantum chemistry contained in the Q-Chem 4 program package. Mol. Phys. 2015, 113, 184-215.

(79) Epifanovsky, E.; Wormit, M.; Kuś, T.; Landau, A.; Zuev, D.; Khistyaev, K.; Manohar, P. U.; Kaliman, I.; Dreuw, A.; Krylov, A. I. New implementation of highlevel correlated methods using a general block-tensor library for high-performance electronic structure calculations. J. Comput. Chem. 2013, 34, 2293-2309.

(80) Stanton, J. F.; Gauss, J.; Cheng, L.; Harding, M. E.; Matthews, D. A.; Szalay, P. G. CFOUR, Coupled-Cluster techniques for Computational Chemistry, a quantumchemical program package. With contributions from A.A. Auer, R.J. Bartlett, U. Benedikt, C. Berger, D.E. Bernholdt, Y.J. Bomble, O. Christiansen, F. Engel, R. Faber, M. Heckert, O. Heun, M. Hilgenberg, C. Huber, T.-C. Jagau, D. Jonsson, J. Jusélius, T. Kirsch, K. Klein, W.J. Lauderdale, F. Lipparini, T. Metzroth, L.A. Mück, D.P. O’Neill, D.R. Price, E. Prochnow, C. Puzzarini, K. Ruud, F. Schiffmann, W. Schwalbach, C. Simmons, S. Stopkowicz, A. Tajti, J. Vázquez, F. Wang, J.D. Watts and the integral packages MOLECULE (J. Almlöf and P.R. Taylor), PROPS (P.R. Taylor), ABACUS (T. Helgaker, H.J. Aa. Jensen, P. Jørgensen, and J. Olsen), and ECP routines by A. V. Mitin and C. van Wüllen. For the current version, see http://www.cfour.de. 
(81) Khani, S. K.; Faber, R.; Santoro, F.; Coriani, S.; Hättig, C. UV Absorption and Magnetic Circular Dichroism Spectra of Purine, Adenine, and Guanine: a Coupled Cluster Study in Vacuo and in Aqueous Solution. J. Chem. Theory Comput. 2019, $15,1242-1254$.

(82) Santoro, F.; Improta, R.; Fahleson, T.; Kauczor, J.; Norman, P.; Coriani, S. Relative Stability of the $\mathrm{L}_{a}$ and $\mathrm{L}_{b}$ Excited States in Adenine and Guanine: Direct Evidence from TD-DFT Calculations of MCD Spectra. J. Phys. Chem. Lett. 2014, 5, 18061811.

(83) Zhang, X.; Herbert, J. M. Excited-State Deactivation Pathways in Uracil versus Hydrated Uracil: Solvatochromatic Shift in the $\ln \pi^{*}$ State is the Key. J. Phys. Chem. B 2014, 118, 7806-7817.

(84) Kaufmann, K.; Baumeister, W.; Jungen, M. Universal Gaussian basis sets for an optimum representation of Rydberg and continuum wavefunctions. J. Phys. B: At., Mol. Opt. Phys. 1989, 22, 2223.

(85) Reisler, H.; Krylov, A. I. Interacting Rydberg and valence states in radicals and molecules: Experimental and theoretical studies. Int. Rev. Phys. Chem. 2009, 28, $267-308$.

(86) Plekan, O.; Feyer, V.; Richter, R.; Coreno, M.; de Simone, M.; Prince, K.; Trofimov, A.; Gromov, E.; Zaytseva, I.; Schirmer, J. A theoretical and experimental study of the near edge X-ray absorption fine structure (NEXAFS) and X-ray photoelectron spectra (XPS) of nucleobases: Thymine and adenine. Chem. Phys. 2008, 347, $360-375$.

(87) Coreno, M.; Avaldi, L.; Camilloni, R.; Prince, K. C.; de Simone, M.; Karvonen, J.; Colle, R.; Simonucci, S. Measurement and ab initio calculation of the Ne photoabsorption spectrum in the region of the $K$ edge. Phys. Rev. A 1999, 59, 2494. 
(88) Schirmer, J.; Trofimov, A.; Randall, K.; Feldhaus, J.; Bradshaw, A. M.; Ma, Y.; Chen, C. T.; Sette, F. K-shell excitation of the water, ammonia and methane molecules using high-resolution photoabsorption spectroscopy. Phys. Rev. A 1993, 47, 1136.

(89) Ma, Y.; Chen, C.; Meigs, G.; Randall, K.; Sette, F. High-resolution K-shell photoabsorption measurements of simple molecules. Phys. Rev. A 1991, 44, 1848.

(90) Püttner, R.; Dominguez, I.; Morgan, T.; Cisneros, C.; Fink, R.; Rotenberg, E.; Warwick, T.; Domke, M.; Kaindl, G.; Schlachter, A. Vibrationally resolved O 1s coreexcitation spectra of CO and NO. Phys. Rev. A 1999, 59, 3415.

(91) McLaren, R.; Clark, S.; Ishii, I.; Hitchcock, A. Absolute oscillator strengths from K-shell electron-energy-loss spectra of the fluoroethenes and 1, 3-perfluorobutadiene. Phys. Rev. A 1987, 36, 1683.

(92) Stranges, S.; Alagia, M.; Fronzoni, G.; Decleva, P. High-Resolution Inner-Shell Photoabsorption and Dissociation of Ozone. J. Phys. Chem. A 2001, 105, 3400-3406.

(93) Feyer, V.; Plekan, O.; Richter, R.; Coreno, M.; de Simone, M.; Prince, K. C.; Trofimov, A. B.; Zaytseva, I. L.; Schirmer, J. Tautomerism in Cytosine and Uracil: A Theoretical and Experimental X-ray Absorption and Resonant Auger Study. J. Phys. Chem. A 2010, 114, 10270-10276.

(94) Rohatgi, A. WebPlotDigitizer, version 4.0, https://automeris.io/WebPlotDigitizer. 2017.

(95) Schaftenaar, G.; Noordik, J. Molden: a pre- and post-processing program for molecular and electronic structures. J. Comput.-Aided Mol. Design 2000, 14, 123-134.

(96) Aidas, K.; Angeli, C.; Bak, K. L.; Bakken, V.; Bast, R.; Boman, L.; Christiansen, O.; Cimiraglia, R.; Coriani, S.; Dahle, P.; Dalskov, E. K.; Ekström, U.; Enevoldsen, T.; Eriksen, J. J.; Ettenhuber, P.; Fernández, B.; Ferrighi, L.; Fliegl, H.; Frediani, L.; 
Hald, K.; Halkier, A.; Hättig, C.; Heiberg, H.; Helgaker, T.; Hennum, A. C.; Hettema, H.; Hjertenæs, E.; Høst, S.; Høyvik, I.-M.; Iozzi, M. F.; Jansik, B.; Jensen, H. J. A.; Jonsson, D.; Jørgensen, P.; Kauczor, J.; Kirpekar, S.; Kjærgaard, T.; Klopper, W.; Knecht, S.; Kobayashi, R.; Koch, H.; Kongsted, J.; Krapp, A.; Kristensen, K.; Ligabue, A.; Lutnæs, O. B.; Melo, J. I.; Mikkelsen, K. V.; Myhre, R. H.; Neiss, C.; Nielsen, C. B.; Norman, P.; Olsen, J.; Olsen, J. M. H.; Osted, A.; Packer, M. J.; Pawlowski, F.; Pedersen, T. B.; Provasi, P. F.; Reine, S.; Rinkevicius, Z.; Ruden, T. A.; Ruud, K.; Rybkin, V. V.; Salek, P.; Samson, C. C. M.; de Merás, A. S.; Saue, T.; Sauer, S. P. A.; Schimmelpfennig, B.; Sneskov, K.; Steindal, A. H.; SylvesterHvid, K. O.; Taylor, P. R.; Teale, A. M.; Tellgren, E. I.; Tew, D. P.; Thorvaldsen, A. J.; Thøgersen, L.; Vahtras, O.; Watson, M. A.; Wilson, D. J. D.; Ziolkowski, M.; Ågren, H. The Dalton Quantum Chemistry Program System. WIREs Comput. Mol. Sci. 2014, 4, 269 .

(97) Jolly, W.; Bomben, K.; Eyermann, C. Core-electron binding energies for gaseous atoms and molecules. Atomic Data and Nuclear Data Tables 1984, 31, 433-493.

(98) Li, Z.; Madjet, M. E.-A.; Vendrell, O.; Santra, R. Core-level transient absorption spectroscopy as a probe of electron hole relaxation in photoionized $\mathrm{H}+\left(\mathrm{H}_{2} \mathrm{O}\right)$ n. Faraday Discuss. 2014, 171, 475.

(99) Bhattacherjee, A.; Pemmaraju, C. D.; Schnorr, K.; Attar, A. R.; Leone, S. R. Ultrafast Intersystem Crossing in Acetylacetone via Femtosecond X-ray Transient Absorption at the Carbon K-Edge. J. Am. Chem. Soc. 2017, 139, 16576-16583.

(100) Neville, S. P.; Averbukh, V.; Patchkovskii, S.; Ruberti, M.; Yun, R.; Chergui, M.; Stolow, A.; Schuurman, M. S. Beyond structure: ultrafast X-ray absorption spectroscopy as a probe of non-adiabatic wavepacket dynamics. Faraday Discuss. 2016, 194, 117-145. 
(101) Neville, S. P.; Averbukh, V.; Ruberti, M.; Yun, R.; Patchkovskii, S.; Chergui, M.; Stolow, A.; Schuurman, M. S. Excited state X-ray absorption spectroscopy: Probing both electronic and structural dynamics. J. Chem. Phys. 2016, 145, 144307.

(102) Neville, S. P.; Chergui, M.; Stolow, A.; Schuurman, M. S. Ultrafast X-Ray Spectroscopy of Conical Intersections. Phys. Rev. Lett. 2018, 120, 243001.

(103) Myhre, R.; Wolf, T.; Cheng, L.; Nandi, S.; Coriani, S.; Gühr, M.; Koch, H. A theoretical and experimental benchmark study of core-excited states in nitrogen. J. Chem. Phys. 2018, 148, 064106.

(104) Liu, J.; Matthews, D.; Coriani, S.; Cheng, L. Benchmark Calculations of K-Edge Ionization Energies for First-Row Elements Using Scalar-Relativistic Core-ValenceSeparated Equation-of-Motion Coupled-Cluster Methods. J. Chem. Theory Comput. 0, o, null, PMID: 30702889. 


\section{Graphical TOC Entry}

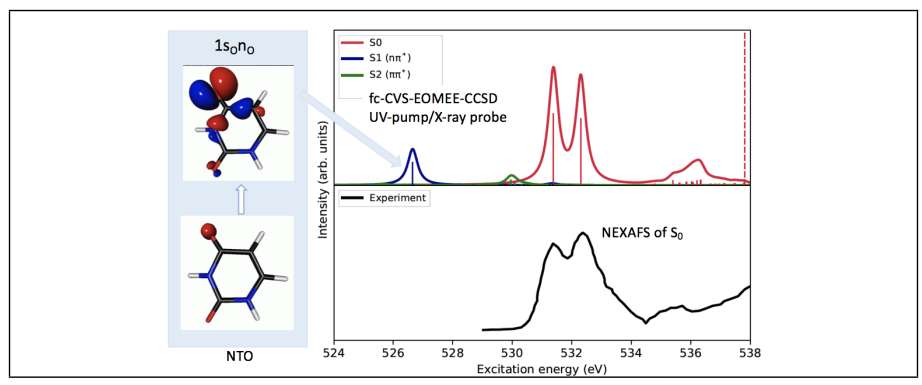

